\title{
Enhancing the productivity of potato crop under drought stress by using some biological treatments
}

\author{
Mostafa M.A.A. Zahran ${ }^{1}$, Mohamed Hefzy ${ }^{2}$ and Hassan H.A. Mostafa ${ }^{3}$
}

\author{
${ }^{1}$ Improvement and Conservation of Cultivated Soils Research Department, Soils, Water and \\ Environment Research Institute (SWERI), Agricultural Research Center (ARC), Giza, Egypt. \\ ${ }^{2}$ Water Requirement and Field Irrigation Research Department, Soils Water and Environment Research \\ Institution, Agricultural Research Center, Giza, Egypt \\ ${ }^{3}$ Central Laboratory of Organic Agriculture, Agricultural Research Centre (Affiliation ID: 60019332), \\ 9 Gamaa Street, 12619 Giza, Egypt \\ Received: 25 Mar. 2020 / Accepted 05 May 2020 / Publication date: 10 May 2020
}

\begin{abstract}
The objectives of current investigation were to investigate the effect of various microbial treatments on the growth and productivity of potato, and nutrient uptake under different irrigation regimes and to study the influence of bacteria to alleviate the negative impact of water deficit irrigation. Therefore, the present study was conducted as a factorial experiment, which the main plot was assigned to various irrigation regimes (100\% of crop evapotranspiration ETc, $80 \%$ ETc and $60 \%$ ETc) and sub-plots were assigned to microbial treatments (control $=\mathrm{ck}$, Azotobacter chroococcum + Azospirillum brasilense $=\mathrm{AZ}$, Bacillus megaterium $=\mathrm{BM}$, Bacillus circulans $=\mathrm{BC}$ and combined $\mathrm{AZ}+\mathrm{BM}+\mathrm{BC}=\mathrm{Mix})$. The main results revealed that the actual amount of water applied was varied according to irrigation regime level and potato growth stage. Increase of water deficit irrigation resulted in a significant decrease in plant height, yield and yield components, dry tubers yield, starch and protein yield, and nitrogen, phosphorus and potassium uptake, however, increased irrigation water use efficiency and economic productivity of irrigation water. Microbial inoculation enhanced all the above studied parameters under various irrigation regimes compared to non-inoculated treatment (control). For the interaction effect, with $20 \%$ water-saving a irrigation regime $80 \%$ ETc, combined microbial treatment the average reduction in both season for tuber weight per plant, marketable yield and tubers total yield were $7.56 \%$, $7.75 \%$ and $7.56 \%$ compared to $100 \%$ ETc, respectively. However, compared to control at $100 \%$ ETc, Mix treatment increased the total yield in both seasons by $19.26 \%$. Moreover, protein and starch yield, dry tubers yield and nitrogen, phosphorus and potassium uptake were higher when potato plants irrigated with $100 \%$ ETc and inoculated with combined microbial treatment.
\end{abstract}

Keywords: Calcareous soil, irrigation regime, microbial treatment, nutrient uptake, tubers yield

\section{Introduction}

The world population is growing and predicted to reach 8.6 billion in 2030 according to the United Nations report (21 June 2017). Consequently, great pressure is placed on farmable land, water, energy and biological resources to provide an adequate supply of food while preserving the integrity of ecosystem. It was well-documented that the loss of arable land due to either salinization or drought has posed a major challenge for maintaining world food supplies for the growing population. In many regions all over the world drought poses one of the most important constraints to plant growth and productivity (Chaves et al., 2003). Moreover, availability of water is becoming even scarcer for agricultural communities. Also, Nadeem et al. (2014) and Delshadi et al. (2017) reported that drought stress has direct effects on the nitrogen fixation amount in plants. In addition, drought stress bothers mineral nutrient linkups, hindering plant growth and evolvement, which reflects on the ultimate crop yield. Drought usually decreases mass flow consequently mineral nutrient uptake and the transmission of these nutrients from the roots to the shoot, influencing in all metabolic activity of plant physiology (Silva et al. 2011; Bista et al., 2018). Therefore, for the next decade, one of the great challenges is to mitigate any effect of climate change on crop production with a main focus being to maintain crop

Corresponding Author: Mohamed Hefzy, Water Requirement and Field Irrigation Research Department, Soils Water and Environment Research Institution, Agricultural Research Center, Giza, Egypt.

E-mail: mhefzy2005@yahoo.com- https://orcid.org/0000-0002-3136-5841 
production levels with reduced availability of water (Obidiegwu et al., 2015). Consequently, it is very important to find an approach to increase the crop tolerance to drought stress.

On the other side, calcareous soils constitute more than $30 \%$ of the world's land surface area, and their $\mathrm{CaCO}_{3}$ content wobbled from a few percent to $95 \%$ (Marschner, 1995). Calcareous soils formed naturally in arid and semiarid regions because of relativity scarce leaching. They also occur in humid and semi humid zones if their parent material is affluent in $\mathrm{CaCO}_{3}$, such as limestone and shells (Brody and Weil, 1999). El-Damaty et al. (1973) suggested 8-10\% $\mathrm{CaCO}_{3}$ as limit for defining calcareous soils. In Egypt, the calcareous soils comprise about $25-30 \%$ of the total area (Taalap et al., 2019). The most fastidious problems for lucrative using of calcareous soils in agriculture are their poor physico-chemical characteristics and comrade poor water retention, and speedily fixation of applied nutrients if added to the soil. Generally, calcareous soils tend to be low in available nitrogen and total organic matter (Wahba et al., 2019). The high pH level (may exceed 9) performs in unavailability of phosphorus, iron, manganese, zinc and copper (Imas and Sheva, 2000). As well as problems of potassium and magnesium nutrition because imbalance between these elements and calcium (Yuncong, 2001). Consequently, chlorosis symptoms are usually observed on plants grown in calcareous soils. But potential productivity may be very high where adequate water and nutrients can be provided (Wahba et al., 2019).

Previous studies reported that application of high nitrogen doses could alleviate the negative effects of water deficit and improve plant growth (Halvorson and Reule 1994; Fife and Nambiar 1997; Van-Schaik et al. 1997; Saneoka et al. 2004). However, the use of chemical fertilizers cause the damage to our ecosystem, atmosphere and harmful to human health (Goyal et al. 2005, Ju et al. 2018); plus it is the greatest input cost for a large number of crops (Amiour et al. 2012). Recently, due to technological advances in next generation sequencing and microbiomics, attention has turned to the application of beneficial microorganisms as biofertilizer, that mediate drought tolerance, improve plant water use efficiency (Jochum et al. 2019) and improve soil properties (Nadeem et al. 2014).

Biofertilizers are products including living cells of distinct types of microbial which when applied to seed, soil or plant surface colonize the rhizosphere or the plant interior. Consequently, enhancement growth by transferring nutritionally serious elements (macro and micro nutrients) from unusable to usable form through different biological process such as nitrogen fixation, solubilization of insoluble phosphate and silicate minerals or potassium releasing (Rokhzadi et al., 2008; Farag Jr et al., 2013; Mamnabi et al., 2020). In addition, microorganisms in biofertilizers support decomposition of organic matter, increasing availability of nutrients, production of antibiotics, phytohormones i.e. IAA, GAs and cytokinin, protect plants from pests and diseases and enhancement crop production (Bhattacharyya and Jha, 2012; Khosro and Yousef, 2012). In this respect, Delshadi et al. (2017) reported that some bacterial species as biofertilizers are widely used to increase plant growth, yield, seed production and biological control in various crops. This could increase nutrient uptake and improves drought tolerance (Suhag (2016) and help the plants to overcome the negative effects of water deficit (Azab 2016).

Potato (Solanum tuberosum L.) is the most important non-cereal food crop worldwide and ranks as the fourth most important food crop and is of great economic value (Pino et al., 2007, Obidiegwu et al., 2015). It is often considered as a drought sensitive crop and its production is threatened due to frequent drought episodes (Obidiegwu et al., 2015). Moreover, potato yield in reclaimed lands are highly profitable and greater than crop yield obtained in the Nile Delta and Nile Valley (Mosa, 2012). Taking into consideration that the experiment soil is calcareous soils, which are widely occurrence in the desert areas of Egypt, it cause some physico-chemical problems that affect crop production, water use efficiency and availability of nutrients in this kind of soil (Abou-Hussien et al., 2019). Consequently, application of Azotobacter chroococcum, Azospirillium brasilense, Bacillus megaterium and Bacillus circulans could be an appropriate method to improve potato yield, yield components and yield quality under water deficit conditions in calcareous soils. However, to the best of our knowledge, the concepts of microbial inoculation and various irrigation regimes have not been fully integrated for potato crop in previous studies.

The structure and chemical composition of potato fraction, such as proteins, starch, organic and inorganic compounds, non-starch polysaccharides, sugars, macro and micro nutrients $(\mathrm{P}, \mathrm{K}, \mathrm{Mg}, \mathrm{Ca}$, $\mathrm{Fe}, \mathrm{Mn}, \mathrm{Zn}$ and $\mathrm{Cu}$ ) influence the quality of potatoes especially for industrial and food purposes products (Ekin, 2011; Dupuis and Qiang 2019). Only after harvest, potato tubers contain about $80 \%$ of water and $20 \%$ of dry matter. Almost 60 to $80 \%$ of the dry matter is starch (Ekin, 2011). Starch is a natural polymer appearing in all plant organisms, an indirect output of photosynthesis, and it has called 
a biodegradable substance (Leszczyński 2004). For potato chips production tubers should contain 14$17 \%$ of starch and $20-22 \%$ of dry matter, while $12-16 \%$ of starch and $18-22 \%$ of dry matter for suitable immediate consumption (Lisińska 2000; Zgórska and Frydecka-Mazurczyk 2002; Baranowska, 2018).

Therefore, the current study aimed to investigate the effect of various microbial treatments on the growth and productivity of potato, and nutrient uptake under different irrigation regimes and to study the influence of bacteria to alleviate the negative impact of water deficit irrigation.

\section{Materials and Methods}

\section{Plant material and experimental site}

The field trials of potato crop (Solanum tuberosum L., cv. Cara) were conducted during two growing seasons, 2017/2018 and 2018/2019 at the Research Farm of Arab El- Awammer Research Station, ARC, Asyut, Egypt (Latitude 27 ${ }^{\circ}, 03^{\prime} \mathrm{N}$ and Longitude 31, $01^{\prime} \mathrm{E}$ and the Altitude $71 \mathrm{~m}$ above sea level). Detailed monthly meteorological data in Asyut during the growth seasons are reported in Table (1). The experiments were conducted in sandy calcareous soil and the classification of soil was Typic Torripsamments agreement with Soil Taxonomy (Soil Survey Staff 2010). Representative soil samples from the field experimental surface layer $(0-25 \mathrm{~cm})$ were collected before cultivation and airdried then crushed, and sifted to pass through $2 \mathrm{~mm}$. The physical and chemical properties were determined by the standard methods reported by Klute (1986) and Jackson (1973), and the obtained results are presented in Table 2 .

Table 1: Average monthly meteorological data of Asyut weather station during the two growth seasons of $2017 / 2018$ and $2018 / 2019$.

\begin{tabular}{lcccccc}
\hline & \multicolumn{7}{c}{$\mathbf{2 0 1 7 / 2 0 1 8}$} \\
\cline { 2 - 4 } & \multicolumn{2}{c}{ Temperature $\left({ }^{\circ} \mathbf{C}\right)$} & $\begin{array}{c}\text { Relative } \\
\text { humidity } \\
\mathbf{( \% )}\end{array}$ & $\begin{array}{c}\text { Wind } \\
\text { speed } \\
\mathbf{( k m / h )}\end{array}$ & $\begin{array}{c}\text { Sunshine } \\
\text { hours (h) }\end{array}$ & ETo (mm) \\
\cline { 2 - 5 } November & 25.1 & 10.9 & 54.6 & 15.2 & 9.4 & 4.75 \\
December & 23.2 & 9.0 & 58.8 & 14.6 & 9.0 & 3.98 \\
January. & 19.9 & 6.5 & 57.5 & 15.3 & 8.9 & 3.73 \\
February. & 26.1 & 11.2 & 44.3 & 14.4 & 9.7 & 5.63 \\
\hline & & & $\mathbf{2 0 1 8 / 2 0 1 9}$ & & & 4.93 \\
November & 26.5 & 13.1 & 53.8 & 14.7 & 9.4 & 3.62 \\
December & 20.8 & 8.0 & 62.8 & 16.3 & 9.0 & 3.70 \\
January. & 19.3 & 5.8 & 52.8 & 13.9 & 8.9 & 4.93 \\
February. & 21.8 & 7.6 & 51.4 & 17.3 & 9.7 & \\
\hline
\end{tabular}

Table 2: Physical and chemical properties of representative composite soil sample from surface layer $(0-25 \mathrm{~cm})$ of the field experimental site.

\begin{tabular}{|c|c|c|c|c|c|c|c|c|c|}
\hline \multicolumn{10}{|c|}{ Chemical properties } \\
\hline \multirow{2}{*}{$\begin{array}{l}\text { pH } \\
(1: 1)\end{array}$} & \multirow{2}{*}{$\begin{array}{l}\text { EC } \\
\text { dS/ m } \\
(1: 1)\end{array}$} & \multicolumn{3}{|c|}{ Soluble cations (meq / L) } & & \multicolumn{2}{|c|}{$\begin{array}{l}\text { Soluble anions } \\
(\mathrm{meq} / \mathrm{L})\end{array}$} & \multirow{2}{*}{$\begin{array}{c}\text { Available } \\
\text { phosphorus } \\
\text { (ppm) }\end{array}$} & \multirow{2}{*}{$\begin{array}{c}\text { Total } \\
\text { nitrogen } \\
(\%)\end{array}$} \\
\hline & & $\mathrm{Ca}^{++}$ & $\mathbf{M g}^{++}$ & $\mathbf{N a}^{+}$ & $\mathbf{K}^{+}$ & $\begin{array}{l}\mathrm{CO}_{3}^{--} \\
+\mathrm{HCO}_{3}^{-}\end{array}$ & $\mathrm{Cl}$ & & \\
\hline 8.44 & 0.41 & 1.59 & 1.25 & 0.38 & 0.78 & 1.89 & 1.64 & 7.01 & 0.01 \\
\hline \multicolumn{10}{|c|}{ Physical properties } \\
\hline \multicolumn{3}{|c|}{$\begin{array}{c}\text { Particle size distribution } \\
(\%)\end{array}$} & Texture & \multicolumn{3}{|c|}{$\begin{array}{l}\text { Moisture content } \\
\text { (Volumetric \%) }\end{array}$} & \multirow{2}{*}{$\begin{array}{l}\text { O.M } \\
(\%)\end{array}$} & \multirow[t]{2}{*}{$\mathrm{CaCO}_{3}(\%)$} & \multirow{2}{*}{$\begin{array}{c}\text { Bulk } \\
\text { density }\end{array}$} \\
\hline Sand & Silt & Clay & & S. $\mathbf{P}$. & F.C. & W.P. & & & \\
\hline 91.1 & 5.7 & 3.2 & Sandy & 23.0 & 10.9 & 4.5 & 0.40 & 29.80 & 1.60 \\
\hline
\end{tabular}

\section{Experimental design}

The present investigation was carried out as a randomized complete block design in a split-plot with three replications. The first factor was assigned in main plot to irrigation regimes 100\% ETc, $80 \%$ ETc and $60 \%$ of crop evapotranspiration ETc). The second factor was assigned in sub plot to microbial treatments (control=ck, Azotobacter chroococcum + Azospirillum brasilense $=\mathrm{AZ}$, Bacillus megaterium 
$=\mathrm{BM}$, Bacillus circulans $=\mathrm{BC}$ and combined $\mathrm{AZ}+\mathrm{BM}+\mathrm{BC}=\mathrm{Mix})$, which provided from Central Lab. of Organic Agriculture, Agricultural Research Centre, Giza, Egypt. The field experiment was conducted under drip irrigation system and the plot area was $20 \mathrm{~m}^{2}$.

\section{Plant growth conditions}

Healthy potato tubers were sown on one side of each dripper line at $30 \mathrm{~cm}$ in between during second week of October in both seasons. During the soil preparation phosphorus was added at the rate of $107.1 \mathrm{~kg} \mathrm{P}_{2} \mathrm{O}_{5} /$ ha in the form of granular superphosphate $\left(15 \% \mathrm{P}_{2} \mathrm{O}_{5}\right)$. Nitrogen fertilizer was added in the form of ammonium nitrate $(33.5 \% \mathrm{~N})$ at the rate of $214.3 \mathrm{~kg} \mathrm{~N} / \mathrm{ha}$ in six equal doses via the irrigation system. However, potassium was applied at the rate of $119 \mathrm{~kg} \mathrm{~K}_{2} \mathrm{O} /$ ha through irrigation system in six equal doses in the form of potassium sulfate $\left(50 \% \mathrm{~K}_{2} \mathrm{O}\right)$. Cheated $\mathrm{Fe}, \mathrm{Mn}$ and $\mathrm{Zn}$ in liquid solution, containing $100 \mathrm{ppm}$ of each was used as foliar spray, sprayed twice. The other agriculture practices were carried out according to the potato extension guide - Ministry of Agriculture, Egypt. At the first week of February irrigation was stopped then potato plants were harvested on March third and second in the first and second seasons, respectively. CROPWAT model was used to calculate reference evapotranspiration with Penman Monteith (Smith, 1991).

Crop evapotranspiration (ETc): (Allen et al. 1998)

$$
E T_{c}=E T_{0} \times K c
$$

Where:-

$\mathrm{ETc}=$ Crop evapotranspiration.

$\mathrm{ET}_{0}=$ Reference evapotranspiration.

$\mathrm{Kc}=$ Crop coefficient (from FAO 56)

The crop coefficient $(\mathrm{Kc})$ for potato growth stages (initial, developmental, middle and tuber maturity stages) was $0.50,0.65,1.15$ and 0.75 , respectively.

Irrigation water applied:-

The amounts of actual irrigation water applied under each irrigation treatment were determined using the following equation: James (1988)

Where:

$$
\text { I. } R a=\frac{E T c+L F}{E r}
$$

I.Ra $=$ total actual irrigation water applied $\mathrm{mm} /$ interval.

$\mathrm{ETc}=$ Crop evapotranspiration using Penman Monteith equation.

$\mathrm{Lf}=$ leaching factor $(10 \%)$.

$\mathrm{Er}=$ irrigation system efficiency $(85 \%)$.

\section{Yield and yield components}

After 10 weeks from cultivation, five plants from each plot were used to measure plant height $(\mathrm{cm})$. After harvesting, tubers fresh weight/plant (g), marketable tubers weight /plant $(\mathrm{g})$, total tuber yield (ton/ha) and marketable tubers yield (ton/ha) were measured. Dry tubers yield (ton/ha) was determined as follow:

$$
\text { Dry tubers yield }(\text { ton } / \text { ha })=\frac{\text { Potato total tubers yield }(\text { ton } / \text { ha }) \times \text { Dry matter }(\%)}{100}
$$

Where, dry matter percentage was determined according to Haque et al. (2018).

\section{Irrigation water use efficiency (IWUE, $\left.\mathrm{kg} / \mathrm{m}^{3}\right)$ :-}

$$
\text { Dry matter }(\%)=\frac{\text { Dry weight }(g)}{\text { Fresh weight }(g)} \times 100
$$

The irrigation water use efficiency (IWUE) values were calculated as follows $\mathrm{kg} / \mathrm{m}^{3}$ :

6. Economic productivity of irrigation water $\left(\mathrm{EPIW}, \$ / \mathrm{m}^{3}\right)$ :

$$
\text { IWUE }(\mathrm{kg} / \mathrm{m} 3)=\frac{\text { Tubers potato yield }(\mathrm{kg} / \mathrm{ha})}{\text { Irrigation Water Applied }(\mathrm{m} 3 / \mathrm{ha})}
$$

EPIW was calculated according to Molden (1997) as follow $\$ / \mathrm{m}^{3}$ : 


$$
\operatorname{EPIW}(\$ / m 3)=\frac{\text { Gross value of product }(\$ / h a)}{\text { Total amount of irrigation applied water }(\mathrm{m} 3 / \mathrm{ha})}
$$

\section{Biochemical measurements}

Five whole potato tubers from each plot were randomly selected and then the fresh weight was recorded. Healthy tubers were washed with running tap water and followed with distilled water. After washing, clean tubers were cut into 2-3 mm slices. Tubers slices were oven-dried at $70{ }^{\circ} \mathrm{C}$ until a constant weight was achieved then the dry weight was determined. Dried tubers were ground to fine powder and accurately $0.5 \mathrm{~g}$ weighted for wet digestion using a mixture of sulfuric acid and hydrogen peroxide (Parkinson and Allen 1975). The digested samples were used to measure nitrogen (N), phosphorus $(\mathrm{P})$ and potassium $(\mathrm{K})$. $\mathrm{N}$ determination was carried out using micro-kjeldahl procedure (Jackson 1973). Total phosphorus was determined coloremetrically by the stannous chloride phosphomolybdic-sulfuric acid method as described by Jackson (1973) and total potassium was measured by the flame photometric method (Page, 1982). Protein and starch yield (kg/ha), and N, P and $\mathrm{K}$ uptakes $(\mathrm{kg} / \mathrm{ha})$ were determined as follows:

Protein yield $(\mathrm{kg} / \mathrm{ha})=$ tubers dry yield $($ ton $/ \mathrm{ha}) \times$ protein $(\%) \times 10$

Where, protein percentage was determined according to Ranganna (1977) as follow:

Protein $(\%)=$ Nitrogen $\% \times 6.25$

Starch yield $(\mathrm{kg} / \mathrm{ha})=$ tuber dry yield $($ ton $/ \mathrm{ha}) \times \operatorname{starch}(\%) \times 10$

Where, starch (\%) was calculated according to Burton (1948) method as follow:

$$
\text { Starch }(\%)=17.457+\{0.891 \times(\text { dry mater } \%-24.182)\}
$$

Nitrogen uptake $(\mathrm{kg} / \mathrm{ha})=$ tubers dry yield $($ ton $/ \mathrm{ha}) \times N \% \times 10$

Phosphorus uptake $(\mathrm{kg} / \mathrm{ha})=$ tubers dry yield $($ ton $/ \mathrm{ha}) \times P \% \times 10$

potassium uptake $(\mathrm{kg} / \mathrm{ha})=$ tubers dry yield $($ ton $/ \mathrm{ha}) \times K \% \times 10$

\section{Data analysis}

All obtained data were subjected to statistical analysis of variance and treatment means were compared for significant differences using the Duncan's multiple range test at $p=0.05$. The MSTATC 2.10 computer program was used to perform all the analysis of variance in accordance with the procedure obtained by Steel and Torrie (1982). Data are presented as the means \pm standard deviations.

\section{Results}

\section{Crop evapotranspiration (ETc, $\mathrm{mm})$}

Results in Fig. 1 indicated that crop evapotranspiration was increased in accordance with plant growth development, which recorded the maximum value $\left(155.7 \mathrm{~mm}\right.$ and $144.1 \mathrm{~mm}$ in the $1^{\text {st }}$ and $2^{\text {nd }}$ seasons, respectively) at the third development stage (Mid-season stage). Then it was decreased at the fourth development stage, which were $76.1 \mathrm{~mm}$ and $76.0 \mathrm{~mm}$ during 2017/2018 and 2018/2019 seasons, respectively.

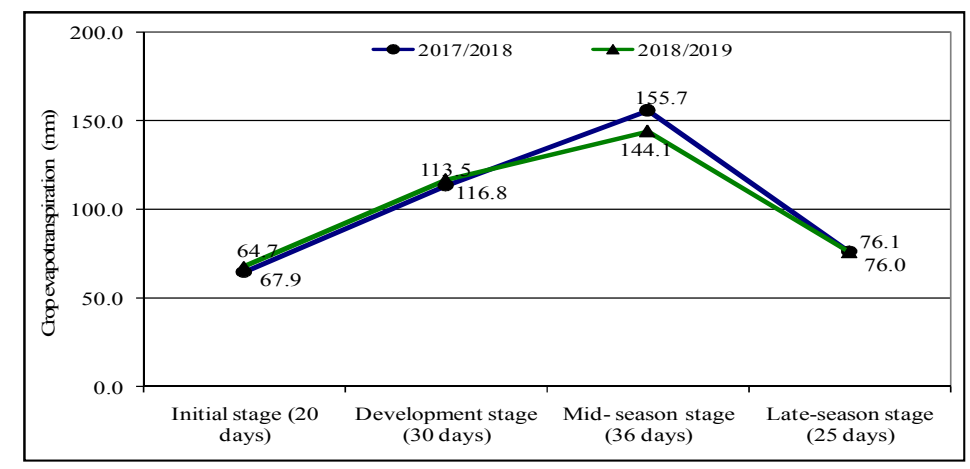

Fig. 1: Changes of crop evapotranspiration ( $\mathrm{mm})$ during various development stages of potato crop during 2017/2018 and 2018/2019 seasons. 


\section{Irrigation water applied $\left(\mathrm{m}^{3} / \mathrm{ha}\right)$}

To know the actual amount of water applied under various irrigation regime during seasons of $2017 / 2018$ and $2018 / 2019$, the irrigation water applied was calculated $\left(\mathrm{m}^{3} / \mathrm{ha}\right)$ at four development stages of potato crop. Data presented in Fig. 2 revealed that the total amount of applied water for potato grown under different irrigation regimes were $5306.0,4244.8$ and $3183.6 \mathrm{~m}^{3} / \mathrm{ha}$ in the first season for $100 \%, 80$ and $60 \%$ ETc, respectively, and were $5208.3,4166.6$ and $3125.0 \mathrm{~m}^{3} /$ ha in the second season. The data in Fig. 2 showed that, the irrigation water applied varies from growth stage to another through the two growth seasons. The lowest value was at the beginning of the growth season. As the plant developed, a gradual increase is observed in water consumption. The irrigation water applied reached its peak at the mid-season stage. After that, the rate of irrigation water applied pronouncedly decreased during the late season of plant growth. The obtained results indicated that the seasonal irrigation water applied was mostly influenced by irrigation treatments.
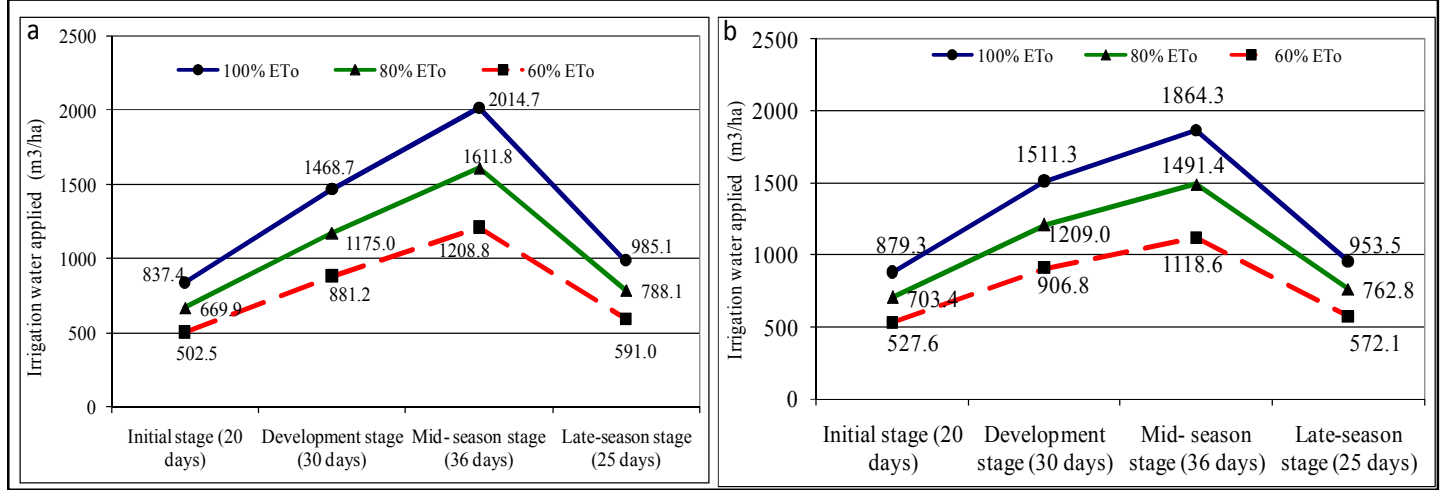

Fig. 2: Irrigation water applied $\left(\mathrm{m}^{3} / \mathrm{ha}\right)$ at different growth stages of potato grown under different irrigation regimes during the seasons of (a) 2017/2018 and (b) 2018/2019

\section{Plant height (cm)}

The height of potato plants in response to various irrigation regimes and biological treatments was measured. As shown in Table 2, irrigation regimes, biological treatments and their interaction significantly affected the plant height of potato $(p<0.01)$. With decreasing irrigation regimes from $100 \% \mathrm{ETc}$ to $60 \% \mathrm{ETc}$, the plant height was significantly decreased during both seasons (from $34.35 \mathrm{~cm}$ and $36.49 \mathrm{~cm}$ for $100 \% \mathrm{ETc}$ to $30.53 \mathrm{~cm}$ and $32.19 \mathrm{~cm}$ for $60 \% \mathrm{ETc}$ in $2017 / 2018$ and $2018 / 2019$ seasons, respectively. Regarding to inoculation treatments, Mix treatment $(\mathrm{AZ}+\mathrm{BM}+\mathrm{BC})$ recorded the longest height of plants $(34.83 \mathrm{~cm}$ and $38.01 \mathrm{~cm}$ ), which was not significantly differ from AZ treatment (Table 3 ). Results of the interaction effect revealed that plants treated with combined microbial (Mix) under irrigation regime $100 \%$ ETc showed highest value of plant height $(36.58 \mathrm{~cm}$ and $40.52 \mathrm{~cm})$. This was not significantly varied from the same inoculation treatment (Mix) under irrigation regime $80 \% \mathrm{ETc}$ in two seasons.

\section{Tubers weight per plant (g)}

To compare the influence of water shortage, inoculation with various bacteria, and their interaction on potato yield, the tubers weight/plant (plant yield) was measured (Table 3). The mean of plant yield (g) was decreased $20.47 \%$ and $20.64 \%$ under irrigation regime $60 \%$ ETc compared to $100 \%$ ETc during two seasons, respectively. Moreover, biological treatments increased significantly the tubers weight per plant compared to un-inoculated plants (control, CK) in both seasons. The highest increase was observed with Mix treatment, which was $30.26 \%$ and $40.31 \%$ compared to control during $1^{\text {st }}$ and $2^{\text {nd }}$ seasons, respectively. Mix treatment under irrigation regime $100 \% \mathrm{ETc}$ gave the biggest mean of plant yield (835.64 g and $926.54 \mathrm{~g}$ in first and second seasons, respectively), which not differ from $\mathrm{BC}$ treatment under $100 \% \mathrm{ETc}$. With saving $20 \%$ of irrigation water, weight of tuber/plant was decreased $7.59 \%$ and $7.53 \%$ under $80 \% \mathrm{ETc}$ and treated plants with $\mathrm{AZ}+\mathrm{BM}+\mathrm{BC}$ (Mix treatment) compared to $100 \% \mathrm{ETc}$ with the same microbial treatment. 
Table 3: Plant height (cm), tubers weight/plant (g), weight of marketable tubers/plant $(\mathrm{g})$ and marketable tubers yield (ton/ha) as influenced by various irrigation regimes and different microbial treatments as well as their interaction.

\begin{tabular}{|c|c|c|c|c|}
\hline \multirow{2}{*}{ Treat. } & \multicolumn{4}{|c|}{$2017 / 2018$} \\
\hline & $100 \%$ ETc & $80 \%$ ETc & $60 \%$ ETc & Mean \\
\hline & \multicolumn{4}{|c|}{ Plant height (cm) } \\
\hline $\mathbf{C K}$ & $32.58 \pm 0.80^{\mathrm{de}}$ & $31.12 \pm 0.38^{\mathrm{fg}}$ & $28.00 \pm 0.66^{\mathrm{h}}$ & $30.57^{\mathrm{c}}$ \\
\hline $\mathbf{A Z}$ & $35.42 \pm 0.58^{\mathrm{b}}$ & $36.00 \pm 0.25^{\mathrm{ab}}$ & $31.82 \pm 0.35^{\mathrm{ef}}$ & $34.41^{\mathrm{a}}$ \\
\hline BM & $33.92 \pm 0.76^{\mathrm{c}}$ & $33.33 \pm 0.63^{\mathrm{cd}}$ & $30.58 \pm 0.14^{\mathrm{g}}$ & $32.61^{\mathrm{b}}$ \\
\hline BC & $33.25 \pm 0.50^{\mathrm{cd}}$ & $33.00 \pm 0.25^{\mathrm{d}}$ & $30.42 \pm 0.14^{\mathrm{g}}$ & $32.22^{\mathrm{b}}$ \\
\hline Mix & $36.58 \pm 0.38^{\mathrm{a}}$ & $36.08 \pm 0.52^{\mathrm{ab}}$ & $31.83 \pm 0.29^{\mathrm{ef}}$ & $34.83^{\mathrm{a}}$ \\
\hline \multirow[t]{2}{*}{ Mean } & $34.35^{\mathrm{a}}$ & $33.91^{\mathrm{a}}$ & $30.53^{\mathrm{b}}$ & \\
\hline & \multicolumn{4}{|c|}{ Tubers weight/plant (g) } \\
\hline CK & $670.29 \pm 32.50^{\mathrm{def}}$ & $611.42 \pm 21.40^{\mathrm{fg}}$ & $453.36 \pm 14.76^{\mathrm{h}}$ & $578.35^{\mathrm{d}}$ \\
\hline $\mathbf{A Z}$ & $676.15 \pm 24.70^{\mathrm{def}}$ & $683.32 \pm 34.02^{\mathrm{de}}$ & $623.78 \pm 38.46^{\mathrm{efg}}$ & $661.08^{c}$ \\
\hline BM & $715.26 \pm 25.71^{\mathrm{cd}}$ & $664.74 \pm 33.10^{\mathrm{def}}$ & $619.70 \pm 28.49^{\mathrm{efg}}$ & $666.57^{\mathrm{c}}$ \\
\hline BC & $792.98 \pm 35.77^{\mathrm{ab}}$ & $754.30 \pm 37.22^{\mathrm{bc}}$ & $588.31 \pm 40.66^{\mathrm{g}}$ & $711.86^{\mathrm{b}}$ \\
\hline Mix & $835.64 \pm 67.75^{\mathrm{a}}$ & $772.18 \pm 31.99^{\mathrm{abc}}$ & $652.30 \pm 29.81^{\mathrm{defg}}$ & $753.37^{\mathrm{a}}$ \\
\hline \multirow[t]{2}{*}{ Mean } & $738.07^{\mathrm{a}}$ & $697.19^{\mathrm{b}}$ & $587.49^{\mathrm{c}}$ & \\
\hline & \multicolumn{4}{|c|}{ Weight of marketable tubers/plant (g) } \\
\hline CK & $511.94 \pm 26.23^{\text {ef }}$ & $455.33 \pm 46.97 \mathrm{~g}$ & $305.92 \pm 10.53^{\mathrm{h}}$ & $424.39^{d}$ \\
\hline $\mathbf{A Z}$ & $635.18 \pm 32.00^{\mathrm{bc}}$ & $553.55 \pm 30.77^{\mathrm{de}}$ & $490.93 \pm 37.94^{\mathrm{fg}}$ & $559.89^{c}$ \\
\hline BM & $592.63 \pm 19.79 \mathrm{~cd}$ & $599.78 \pm 24.13^{\mathrm{cd}}$ & $527.05 \pm 45.32^{\mathrm{ef}}$ & $573.15^{\mathrm{c}}$ \\
\hline $\mathbf{B C}$ & $682.75 \pm 10.55^{\mathrm{ab}}$ & $609.73 \pm 42.35^{\mathrm{cd}}$ & $529.86 \pm 24.45^{\mathrm{ef}}$ & $607.45^{\mathrm{b}}$ \\
\hline Mix & $707.62 \pm 27.05^{\mathrm{a}}$ & $652.53 \pm 29.56^{\mathrm{abc}}$ & $610.09 \pm 40.84^{\mathrm{cd}}$ & $656.75^{\mathrm{a}}$ \\
\hline \multirow[t]{2}{*}{ Mean } & $626.02^{\mathrm{a}}$ & $574.18^{\mathrm{b}}$ & $492.77^{\mathrm{c}}$ & \\
\hline & \multicolumn{4}{|c|}{ Marketable tubers yield (ton/ha) } \\
\hline $\mathbf{C K}$ & $20.469 \pm 1.05^{\mathrm{ef}}$ & $18.206 \pm 1.88^{\mathrm{g}}$ & $12.232 \pm 0.42^{\mathrm{h}}$ & $16.969^{\mathrm{d}}$ \\
\hline $\mathbf{A Z}$ & $25.397 \pm 1.28^{\mathrm{bc}}$ & $22.133 \pm 1.23^{\mathrm{de}}$ & $19.629 \pm 1.52^{\mathrm{fg}}$ & $22.387^{\mathrm{c}}$ \\
\hline BM & $23.696 \pm 0.79^{\mathrm{cd}}$ & $23.982 \pm 0.96^{\mathrm{cd}}$ & $21.074 \pm 1.81^{\mathrm{ef}}$ & $22.917^{\mathrm{c}}$ \\
\hline BC & $27.299 \pm 0.42^{\mathrm{ab}}$ & $24.380 \pm 1.69^{\mathrm{cd}}$ & $21.186 \pm 0.98^{\mathrm{ef}}$ & $24.288^{\mathrm{b}}$ \\
\hline Mix & $28.294 \pm 1.08^{\mathrm{a}}$ & $26.091 \pm 1.18^{\mathrm{abc}}$ & $24.394 \pm 1.63^{\mathrm{cd}}$ & $26.259^{\mathrm{a}}$ \\
\hline \multirow[t]{3}{*}{ Mean } & $25.031^{\mathrm{a}}$ & $22.958^{\mathrm{b}}$ & $19.703^{c}$ & \\
\hline & \multicolumn{4}{|c|}{$2018 / 2019$} \\
\hline & \multicolumn{4}{|c|}{ Plant height $(\mathrm{cm})$} \\
\hline $\mathbf{C K}$ & $34.18 \pm 0.94^{\mathrm{b}}$ & $33.56 \pm 0.70^{\mathrm{b}}$ & $29.80 \pm 0.32^{\mathrm{d}}$ & $32.51^{\mathrm{c}}$ \\
\hline $\mathbf{A Z}$ & $39.20 \pm 0.29^{\mathrm{a}}$ & $39.19 \pm 0.88^{a}$ & $34.00 \pm 1.37^{\mathrm{b}}$ & $37.46^{\mathrm{a}}$ \\
\hline BM & $34.98 \pm 0.44^{\mathrm{b}}$ & $34.67 \pm 0.69^{\mathrm{b}}$ & $31.98 \pm 0.74^{\mathrm{c}}$ & $33.87^{\mathrm{b}}$ \\
\hline BC & $33.58 \pm 0.88^{b}$ & $33.63 \pm 0.95^{\mathrm{b}}$ & $31.13 \pm 0.74^{\mathrm{cd}}$ & $32.78^{c}$ \\
\hline Mix & $40.52 \pm 0.60^{\mathrm{a}}$ & $39.48 \pm 0.86^{\mathrm{a}}$ & $34.05 \pm 0.45^{\mathrm{b}}$ & $38.01^{\mathrm{a}}$ \\
\hline \multirow[t]{2}{*}{ Mean } & $36.49^{\mathrm{a}}$ & $36.11^{\mathrm{a}}$ & $32.19^{\mathrm{b}}$ & \\
\hline & \multicolumn{4}{|c|}{ Tubers weight/plant (g) } \\
\hline $\mathbf{C K}$ & $694.65 \pm 23.16^{\mathrm{ef}}$ & $629.89 \pm 21.97 \mathrm{~g}$ & $463.00 \pm 9.91^{\mathrm{h}}$ & $595.85^{\mathrm{e}}$ \\
\hline $\mathbf{A Z}$ & $774.93 \pm 36.38^{\mathrm{cd}}$ & $692.32 \pm 16.89^{\mathrm{ef}}$ & $643.49 \pm 30.77^{\mathrm{fg}}$ & $703.58^{d}$ \\
\hline BM & $794.12 \pm 27.43^{c}$ & $721.55 \pm 29.47^{\mathrm{e}}$ & $689.00 \pm 25.56^{\mathrm{ef}}$ & $734.89^{c}$ \\
\hline BC & $879.62 \pm 27.02^{\mathrm{ab}}$ & $793.73 \pm 30.91^{\mathrm{c}}$ & $709.47 \pm 51.12^{\mathrm{e}}$ & $794.27^{\mathrm{b}}$ \\
\hline Mix & $926.54 \pm 64.09^{\mathrm{a}}$ & $856.73 \pm 27.74^{b}$ & $724.86 \pm 26.19^{\mathrm{de}}$ & $836.04^{\mathrm{a}}$ \\
\hline \multirow[t]{2}{*}{ Mean } & $813.97^{\mathrm{a}}$ & $738.84^{b}$ & $645.96^{\mathrm{c}}$ & \\
\hline & \multicolumn{4}{|c|}{ Weight of marketable tubers/plant (g) } \\
\hline $\mathbf{C K}$ & $575.46 \pm 17.48^{f}$ & $499.86 \pm 25.02^{\mathrm{g}}$ & $348.84 \pm 20.47^{\mathrm{h}}$ & $474.72^{\mathrm{c}}$ \\
\hline $\mathbf{A Z}$ & $647.70 \pm 13.14^{\mathrm{de}}$ & $673.24 \pm 23.31^{\mathrm{cd}}$ & $566.02 \pm 47.29^{f}$ & $628.99^{\mathrm{b}}$ \\
\hline BM & $672.56 \pm 35.91^{\mathrm{cd}}$ & $667.09 \pm 17.36^{\mathrm{cd}}$ & $587.09 \pm 51.09^{\mathrm{ef}}$ & $642.25^{\mathrm{b}}$ \\
\hline BC & $758.36 \pm 2.74^{\mathrm{ab}}$ & $714.71 \pm 64.54^{\mathrm{bc}}$ & $637.84 \pm 26.90^{\text {de }}$ & $703.64^{\mathrm{a}}$ \\
\hline Mix & $785.72 \pm 34.65^{\mathrm{a}}$ & $725.11 \pm 24.82^{\mathrm{abc}}$ & $678.43 \pm 50.85^{\mathrm{cd}}$ & $729.75^{\mathrm{a}}$ \\
\hline \multirow[t]{2}{*}{ Mean } & $687.96^{\mathrm{a}}$ & $656.00^{\mathrm{b}}$ & $563.65^{\mathrm{c}}$ & \\
\hline & & Marketable & ield (ton/ha) & \\
\hline $\mathbf{C K}$ & $23.009 \pm 0.70^{\mathrm{f}}$ & $19.987 \pm 1.00^{\mathrm{g}}$ & $13.948 \pm 0.82^{\mathrm{h}}$ & $18.981^{\mathrm{c}}$ \\
\hline $\mathbf{A Z}$ & $25.898 \pm 0.53^{\mathrm{de}}$ & $26.919 \pm 0.93^{\mathrm{cd}}$ & $22.632 \pm 1.89^{f}$ & $25.150^{\mathrm{b}}$ \\
\hline BM & $26.892 \pm 1.44^{\mathrm{cd}}$ & $26.673 \pm 0.69^{\mathrm{cd}}$ & $23.474 \pm 2.04^{\mathrm{ef}}$ & $25.680^{\mathrm{b}}$ \\
\hline BC & $30.322 \pm 0.11^{\mathrm{ab}}$ & $28.577 \pm 2.58^{\mathrm{bc}}$ & $25.504 \pm 1.08^{\mathrm{de}}$ & $28.134^{\mathrm{a}}$ \\
\hline Mix & $31.416 \pm 1.39^{\mathrm{a}}$ & $28.993 \pm 0.99^{\mathrm{abc}}$ & $27.126 \pm 2.03^{\mathrm{cd}}$ & $29.178^{\mathrm{a}}$ \\
\hline Mean & $27.507^{\mathrm{a}}$ & $26.230^{\mathrm{b}}$ & $22.537^{\mathrm{c}}$ & \\
\hline
\end{tabular}

Values are given as the mean \pm standard deviation. Different letters indicate statistically differences according to Duncan's multiple range tests.

CK: Control, AZ: Azotobacter chroococcum + Azospirillum brasilense, BM: Bacillus megaterium, BC: Bacillus circulans Mix: Combined $\mathrm{AZ}+\mathrm{BM}+\mathrm{BC}$ ). 


\section{Weight of marketable tubers/plant (g)}

Marketable tubers weight per plant for various microbial treatments at different irrigation regimes during 2017/2018 and 2018/2019 seasons is presented in Table 3. The greatest value of marketable tubers per plant was obtained at $100 \% \mathrm{ETc}\left(626.02 \mathrm{~g}\right.$ and $687.96 \mathrm{~g}$ for $1^{\text {st }}$ and $2^{\text {nd }}$ seasons, respectively). This was followed by $80 \% \mathrm{ETc}$ with 8.28 and 4.65 reduction percentage compared to $100 \% \mathrm{ETc}$. Compared with control, all microbial treatments increased fresh marketable tubers weight per plant during both seasons. Mix treatment showed the maximum weight of marketable tubers per plant $(656.75 \mathrm{~g}$ and $729.75 \mathrm{~g})$ over $\mathrm{CK}$ and other microbial treatments. Potato plants inoculated with $\mathrm{AZ}+\mathrm{BM}+\mathrm{BC}$ (Mix treatment) and received $80 \%$ of ETc showed a significant improvement over control at $100 \% \mathrm{ETc}$ on the marketable tubers weight/plant by $27.46 \%$ and $26.01 \%$ during both seasons.

\section{Marketable tubers yield (ton/ha)}

Water deficits decreased significantly marketable tubers yield (ton/ha) compared to the irrigation with $100 \%$ ETc (Table 3). The greatest marketable tubers yield reduction was recorded at $60 \%$ ETc (21.29\% and $18.07 \%$ during 2017/2018 and 2018/2019 seasons, respectively). However, microbial treatments showed an improvement effect on yield of marketable tuber compared with control at various irrigation regimes. Mix treatment increased marketable tubers yield by $54.75 \%$ and $53.72 \%$ in $1^{\text {st }}$ and $2^{\text {nd }}$ seasons, respectively. The result of interaction effects showed that inoculated potato plants with $\mathrm{AZ}+\mathrm{BM}+\mathrm{BC}$ combined at irrigation regime $100 \%$ ETc recorded greatest yield of marketable tubers (28.294 and 31.416 ton/ha during first and second seasons, respectively). The same treatment (Mix treatment) at $80 \%$ ETc recorded $7.79 \%$ and $7.71 \%$ reduction compared with $100 \%$ ETc in both seasons. However, marketable tubers yield increased by $27.47 \%$ and $26.01 \%$ compared to control at $100 \%$ ETc.

\section{Total yield (ton/ha)}

Various irrigation regimes and inoculation treatments, as well as their interactions, significantly affected the potato total yield (ton/ha) at $p<0.01$ during both seasons (Table 4). Irrigation regime $100 \%$ ETc showed greater average yield (29.511 and 32.546 ton/ha). Compared to $100 \%$ ETc, $80 \%$ ETc reduced total yield by $5.54 \%$ and $9.23 \%$ in 2017/2018 and 2018/2019 seasons, respectively. Moreover, microbial treatments significantly increased the total yield compared with control treatment over all irrigation regimes. More specifically, Mix treatment increased potato total yield by $30.26 \%$ and $40.31 \%$ compared to control. According to interaction effects of irrigation regimes and microbial inoculation, there was no significant different between Mix treatment at irrigation regime $60 \%$ ETc and control plants at $100 \%$ ETc during two seasons. Furthermore, with water saving up to $20 \%$, irrigation regime $80 \%$ ETc with Mix treatment recorded only $7.59 \%$ and $7.53 \%$ reduction percentage for potato total yield compared with the same treatment at $100 \%$ ETc. Meanwhile, increased total yield by $15.20 \%$ and $23.33 \%$ compared to un-inoculated treatment $(\mathrm{CK})$ at $100 \% \mathrm{ETc}$ in the $1^{\text {st }}$ and $2^{\text {nd }}$ seasons, respectively.

\section{Irrigation water use efficiency (IWUE, $\mathrm{Kg} / \mathrm{m}^{3}$ )}

Irrigation water use efficiency for tubers potato was obtained from the tubers potato values divided by the values of irrigation water applied $\left(\mathrm{m}^{3} / \mathrm{ha}\right)$. The results illustrated in Table 4 showed that the lowest values of IWUE were 5.05 and $5.33 \mathrm{~kg} / \mathrm{m}^{3}$ at control under irrigation with $100 \% \mathrm{ETc}$, while the highest $\left(8.19\right.$ and $\left.9.27 \mathrm{~kg} / \mathrm{m}^{3}\right)$ values were obtained at application of combined bacteria $(\mathrm{AZ}+\mathrm{BM}+\mathrm{BC})$ under irrigation with $60 \% \mathrm{ETc}$, in the first and second seasons, respectively. The above results showed that bacterial inoculums application increased irrigation water use efficiency at various irrigation regimes compared to control treatment.

\section{Economic productivity of irrigation water (EPIW, $\left.\$ / \mathrm{m}^{3}\right)$}

Economic productivity of irrigation water for tubers potato was achieved from the gross value of product $(\$ / \mathrm{ha})$ of potato tubers divided by the values of irrigation water applied $\left(\mathrm{m}^{3} / \mathrm{ha}\right)$. Different irrigation regimes, inoculation treatments and their interactions significantly affected the potato EPIW $\left(\$ / \mathrm{m}^{3}\right)$ at $p<0.01$ during both seasons (Table 4). In addition, the trend of EPIW was similar to the IWUE for tubers potato in both seasons. The results reflect that the lowest values of EPIW were 1.26 and 1.33 $\$ / \mathrm{m}^{3}$ at control under irrigation with $100 \%$ ETc, while the highest $\left(2.05\right.$ and $\left.2.32 \$ / \mathrm{m}^{3}\right)$ values were recorded at utilization of conjoined microbial $(\mathrm{AZ}+\mathrm{BM}+\mathrm{BC})$ under irrigation with $60 \% \mathrm{ETc}$, in the first and second seasons, respectively. The indicated results declared that microbial inoculums 
utilization enhanced economic productivity of irrigation water at different irrigation regimes compared to un-inoculated treatment (CK).

Table 4: Influence of different irrigation regimes, microbial inoculation and their interaction on total yield (ton/ha), irrigation water use efficiency $\left(\mathrm{kg} / \mathrm{m}^{3}\right)$ and water productivity $\left(\$ / \mathrm{m}^{3}\right)$.

\begin{tabular}{|c|c|c|c|c|}
\hline \multirow{2}{*}{ Treat. } & \multicolumn{4}{|c|}{ 2017/2018 } \\
\hline & $100 \%$ ETc & $80 \%$ ETc & $60 \%$ ETc & Mean \\
\hline & \multicolumn{4}{|c|}{ Total yield (ton/ha) } \\
\hline CK & $26.801 \pm 1.30^{\mathrm{def}}$ & $24.447 \pm 0.86^{\mathrm{fg}}$ & $18.127 \pm 0.59^{\mathrm{h}}$ & $23.125^{\mathrm{d}}$ \\
\hline $\mathbf{A Z}$ & $27.035 \pm 0.99^{\mathrm{def}}$ & $27.322 \pm 1.36^{\mathrm{de}}$ & $24.941 \pm 1.54^{\mathrm{efg}}$ & $26.433^{c}$ \\
\hline BM & $28.599 \pm 1.03^{\mathrm{cd}}$ & $26.579 \pm 1.32^{\text {def }}$ & $24.778 \pm 1.14^{\text {efg }}$ & $26.652^{\mathrm{c}}$ \\
\hline BC & $31.707 \pm 1.43^{\mathrm{ab}}$ & $30.160 \pm 1.49^{\mathrm{bc}}$ & $23.523 \pm 1.63^{\mathrm{g}}$ & $28.463^{b}$ \\
\hline Mix & $33.412 \pm 2.71^{\mathrm{a}}$ & $30.875 \pm 1.28^{\mathrm{abc}}$ & $26.081 \pm 1.19^{\text {defg }}$ & $30.123^{\mathrm{a}}$ \\
\hline \multirow[t]{2}{*}{ Mean } & $29.511^{\mathrm{a}}$ & $27.876^{\mathrm{b}}$ & $23.490^{\mathrm{c}}$ & \\
\hline & \multicolumn{4}{|c|}{ Irrigation water use efficiency $\left(\mathrm{Kg} / \mathrm{m}^{3}\right)$} \\
\hline CK & $5.05 \pm 0.24^{\mathrm{g}}$ & $5.76 \pm 0.20^{\mathrm{ef}}$ & $5.70 \pm 0.19^{\text {efg }}$ & $5.50^{\mathrm{d}}$ \\
\hline $\mathbf{A Z}$ & $5.10 \pm 0.19^{\mathrm{g}}$ & $6.43 \pm 0.32^{\mathrm{d}}$ & $7.83 \pm 0.48^{\mathrm{ab}}$ & $6.45^{\mathrm{c}}$ \\
\hline BM & $5.39 \pm 0.19^{\mathrm{fg}}$ & $6.26 \pm 0.31^{\mathrm{de}}$ & $7.78 \pm 0.36^{\mathrm{ab}}$ & $6.48^{\mathrm{c}}$ \\
\hline BC & $5.97 \pm 0.27^{\mathrm{def}}$ & $7.10 \pm 0.35^{\mathrm{c}}$ & $7.39 \pm 0.51^{\mathrm{bc}}$ & $6.82^{\mathrm{b}}$ \\
\hline Mix & $6.30 \pm 0.51^{\text {de }}$ & $7.27 \pm 0.30^{\mathrm{bc}}$ & $8.19 \pm 0.37^{\mathrm{a}}$ & $7.26^{\mathrm{a}}$ \\
\hline \multirow[t]{2}{*}{ Mean } & $5.56^{\mathrm{c}}$ & $6.57^{\mathrm{b}}$ & $7.38^{\mathrm{a}}$ & \\
\hline & \multicolumn{4}{|c|}{ Economic productivity of irrigation water (EPIW, $\left.\$ / \mathbf{m}^{3}\right)$} \\
\hline $\mathbf{C K}$ & $1.26 \pm 0.06^{\mathrm{g}}$ & $1.44 \pm 0.05^{\text {ef }}$ & $1.42 \pm 0.05^{\text {efg }}$ & $1.37^{\mathrm{d}}$ \\
\hline $\mathbf{A Z}$ & $1.27 \pm 0.05^{\mathrm{g}}$ & $1.61 \pm 0.08^{\mathrm{d}}$ & $1.96 \pm 0.12^{\mathrm{ab}}$ & $1.61^{\mathrm{c}}$ \\
\hline BM & $1.35 \pm 0.05^{\mathrm{fg}}$ & $1.57 \pm 0.08^{\mathrm{de}}$ & $1.95 \pm 0.09^{\mathrm{ab}}$ & $1.62^{\mathrm{c}}$ \\
\hline BC & $1.49 \pm 0.07^{\mathrm{de}} \mathrm{f}$ & $1.78 \pm 0.09^{\mathrm{c}}$ & $1.85 \pm 0.13^{\mathrm{bc}}$ & $1.71^{\mathrm{b}}$ \\
\hline Mix & $1.57 \pm 0.13^{\mathrm{de}}$ & $1.82 \pm 0.08^{\mathrm{bc}}$ & $2.05 \pm 0.09^{\mathrm{a}}$ & $1.81^{\mathrm{a}}$ \\
\hline \multirow[t]{3}{*}{ Mean } & $1.39^{\mathrm{c}}$ & $1.64^{\mathrm{b}}$ & $1.84^{\mathrm{a}}$ & \\
\hline & \multicolumn{4}{|c|}{ 2018/2019 } \\
\hline & \multicolumn{4}{|c|}{ Total yield (ton/ha) } \\
\hline $\mathbf{C K}$ & $27.775 \pm 0.93^{\text {ef }}$ & $25.186 \pm 0.88^{\mathrm{g}}$ & $18.513 \pm 0.40^{\mathrm{h}}$ & $23.824^{\mathrm{e}}$ \\
\hline $\mathbf{A Z}$ & $30.985 \pm 1.45^{\mathrm{cd}}$ & $27.682 \pm 0.68^{\mathrm{ef}}$ & $25.729 \pm 1.23^{\mathrm{fg}}$ & $28.132^{\mathrm{d}}$ \\
\hline BM & $31.752 \pm 1.10^{\mathrm{c}}$ & $28.851 \pm 1.18^{\mathrm{e}}$ & $27.549 \pm 1.02^{\mathrm{ef}}$ & $29.384^{\mathrm{c}}$ \\
\hline BC & $35.171 \pm 1.08^{\mathrm{ab}}$ & $31.736 \pm 1.24^{\mathrm{c}}$ & $28.367 \pm 2.04^{\mathrm{e}}$ & $31.758^{\mathrm{b}}$ \\
\hline Mix & $37.047 \pm 2.56^{\mathrm{a}}$ & $34.256 \pm 1.11^{\mathrm{b}}$ & $28.983 \pm 1.05^{\mathrm{de}}$ & $33.428^{\mathrm{a}}$ \\
\hline \multirow[t]{2}{*}{ Mean } & $32.546^{\mathrm{a}}$ & $29.542^{\mathrm{b}}$ & $25.828^{\mathrm{c}}$ & \\
\hline & \multicolumn{4}{|c|}{ Irrigation water use efficiency $\left(\mathrm{Kg} / \mathrm{m}^{3}\right)$} \\
\hline $\mathbf{C K}$ & $5.33 \pm 0.18^{\mathrm{f}}$ & $6.04 \pm 0.21^{\mathrm{e}}$ & $5.92 \pm 0.13^{\mathrm{e}}$ & $5.77^{\mathrm{e}}$ \\
\hline $\mathbf{A Z}$ & $5.95 \pm 0.28^{\mathrm{e}}$ & $6.64 \pm 0.16^{\mathrm{d}}$ & $8.23 \pm 0.39^{\mathrm{b}}$ & $6.94^{\mathrm{d}}$ \\
\hline BM & $6.10 \pm 0.21^{\mathrm{e}}$ & $6.92 \pm 0.28^{\mathrm{d}}$ & $8.82 \pm 0.33^{\mathrm{a}}$ & $7.28^{\mathrm{c}}$ \\
\hline BC & $6.75 \pm 0.21^{\mathrm{d}}$ & $7.62 \pm 0.30^{\mathrm{c}}$ & $9.08 \pm 0.65^{\mathrm{a}}$ & $7.82^{\mathrm{b}}$ \\
\hline Mix & $7.11 \pm 0.49^{\mathrm{cd}}$ & $8.22 \pm 0.27^{\mathrm{b}}$ & $9.27 \pm 0.34^{\mathrm{a}}$ & $8.20^{\mathrm{a}}$ \\
\hline \multirow[t]{2}{*}{ Mean } & $6.25^{\mathrm{c}}$ & $7.09^{\mathrm{b}}$ & $8.27^{\mathrm{a}}$ & \\
\hline & \multicolumn{4}{|c|}{ Economic productivity of irrigation water $\left(\mathrm{EPIW}, \$ / \mathrm{m}^{3}\right)$} \\
\hline $\mathbf{C K}$ & $1.33 \pm 0.04^{\mathrm{f}}$ & $1.51 \pm 0.05^{\mathrm{e}}$ & $1.48 \pm 0.03^{\mathrm{e}}$ & $1.44^{\mathrm{e}}$ \\
\hline $\mathbf{A Z}$ & $1.49 \pm 0.07^{\mathrm{e}}$ & $1.66 \pm 0.04^{\mathrm{d}}$ & $2.06 \pm 0.10^{\mathrm{b}}$ & $1.74^{\mathrm{d}}$ \\
\hline BM & $1.52 \pm 0.05^{\mathrm{e}}$ & $1.73 \pm 0.07^{\mathrm{d}}$ & $2.20 \pm 0.08^{\mathrm{a}}$ & $1.82^{\mathrm{c}}$ \\
\hline BC & $1.69 \pm 0.05^{\mathrm{d}}$ & $1.90 \pm 0.07^{\mathrm{c}}$ & $2.27 \pm 0.16^{\mathrm{a}}$ & $1.95^{\mathrm{b}}$ \\
\hline Mix & $1.78 \pm 0.12^{\mathrm{cd}}$ & $2.06 \pm 0.07^{\mathrm{b}}$ & $2.32 \pm 0.08^{\mathrm{a}}$ & $2.05^{\mathrm{a}}$ \\
\hline Mean & $1.56^{\mathrm{c}}$ & $1.77^{\mathrm{b}}$ & $2.07^{\mathrm{a}}$ & \\
\hline
\end{tabular}

Values are given as the mean \pm standard deviation and different letters indicate statistically differences according to Duncan's multiple range tests.

CK: Control, AZ: Azotobacter chroococcum + Azospirillum brasilense, BM: Bacillus megaterium, BC: Bacillus circulans Mix: Combined $\mathrm{AZ}+\mathrm{BM}+\mathrm{BC})$.

\section{Protein yield (kg/ha)}

There were highly significant differences among irrigation regimes and microbial treatments as well as their interaction effects in the yield of protein (Fig. 3). In total, the maximum protein yield (428.27 and $511.01 \mathrm{~kg} / \mathrm{ha}$ during first and second seasons, respectively) was produced from irrigation 
regime $100 \%$ ETc and decreased with increasing water deficit. Moreover, the results showed that inoculation treatments increased significantly protein yield compared to control in both seasons. The highest protein yield was obtained from potato plants subjected to Mix treatment (399.92 and 482.96 $\mathrm{kg}$ /ha during 2017/2018 and 2018/2019 seasons, respectively). On the other hand, control treatments recorded only, 289.41 and $320 \mathrm{~kg} / \mathrm{ha}$ during $1^{\text {st }}$ and $2^{\text {nd }}$ seasons, respectively. According to the interaction effects, highest protein yield $(477.24$ and $572.15 \mathrm{~kg} / \mathrm{ha})$ in both seasons was recorded when potato plants watered with $100 \% \mathrm{ETc}$ and inoculated with combined treatment $(\mathrm{AZ}+\mathrm{BM}+\mathrm{BC})$ with no significant difference with inoculation with $\mathrm{BC}$ in two seasons.

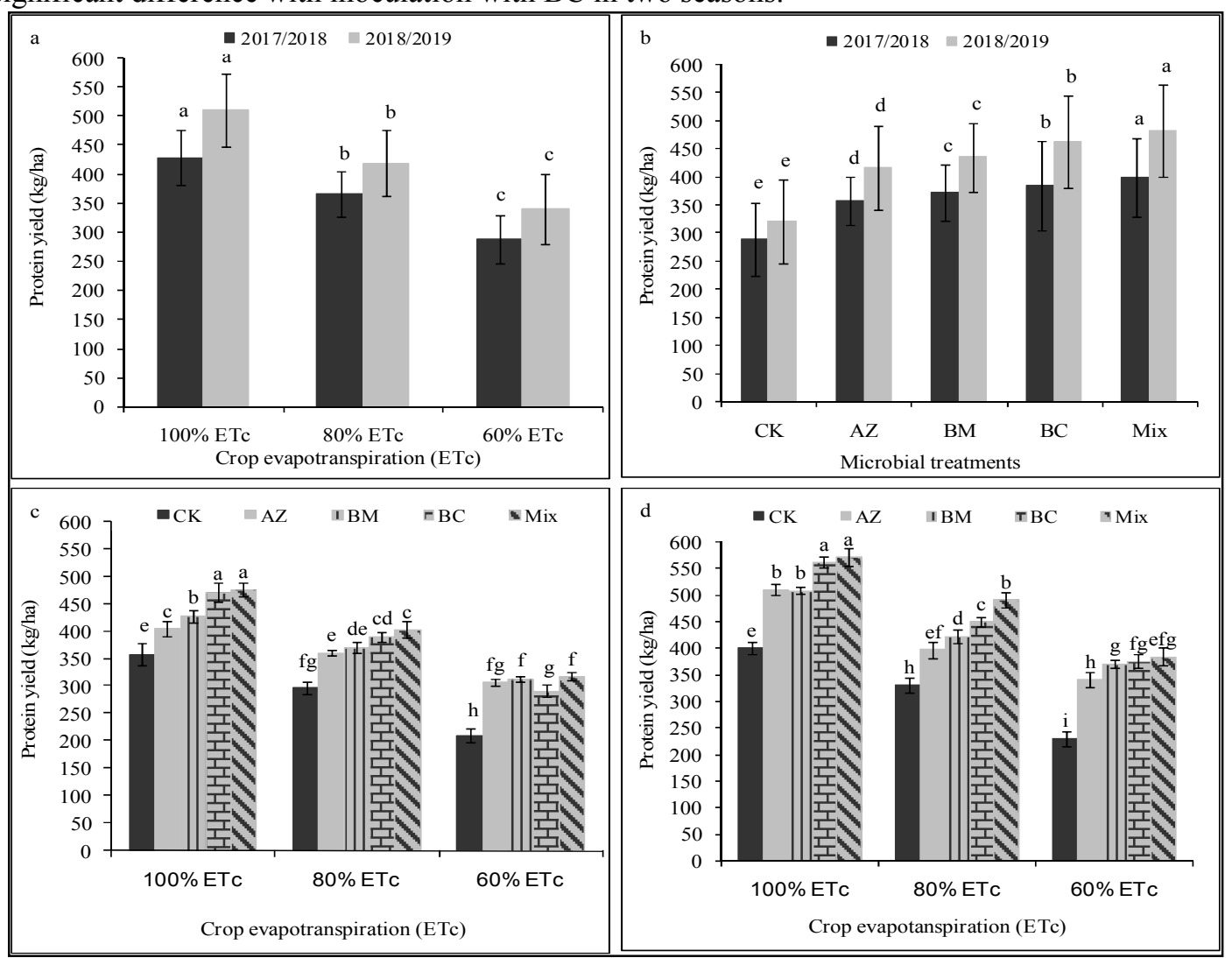

Fig. 3: Influence of irrigation regimes (a), microbial treatments (b) and their interaction during $2017 / 2018$ (c) and 2018/2019 (d) seasons on the protein yield (kg/ha). Data are presented as means \pm SDs and the different upper letters indicate significant differences at $P<0.05$ level according to Duncan's multiple range tests, $(n=15$ in irrigation regimes treatments, $n=9$ in microbial treatments and $\mathrm{n}=3$ in their interaction treatments).

\section{Starch yield (kg/ha)}

The results showed a highly significant difference on the starch yield regarding the effect of various irrigation regimes and microbial treatments (Fig. 4). There was a decrease in starch yield with increased imposing of water deficit. Furthermore, inoculation treatment enhanced the production of starch and alleviated the negative impact of water shortage on starch synthesis compared to untreated plants with bacteria. The starch reduction percentage in control treatment ranged from $20.75 \%$ compared to AZ treatment to $31.05 \%$ compared to Mix treatment in the first season and ranged from $25.05 \%$ to $37.22 \%$ in second season compared to same treatments, respectively. Moreover, Mix treatment under irrigation regime 100\% ETc showed the highest starch yield in two seasons (654.79 and $775.72 \mathrm{~kg} /$ ha during $1^{\text {st }}$ and $2^{\text {nd }}$ seasons, respectively). 


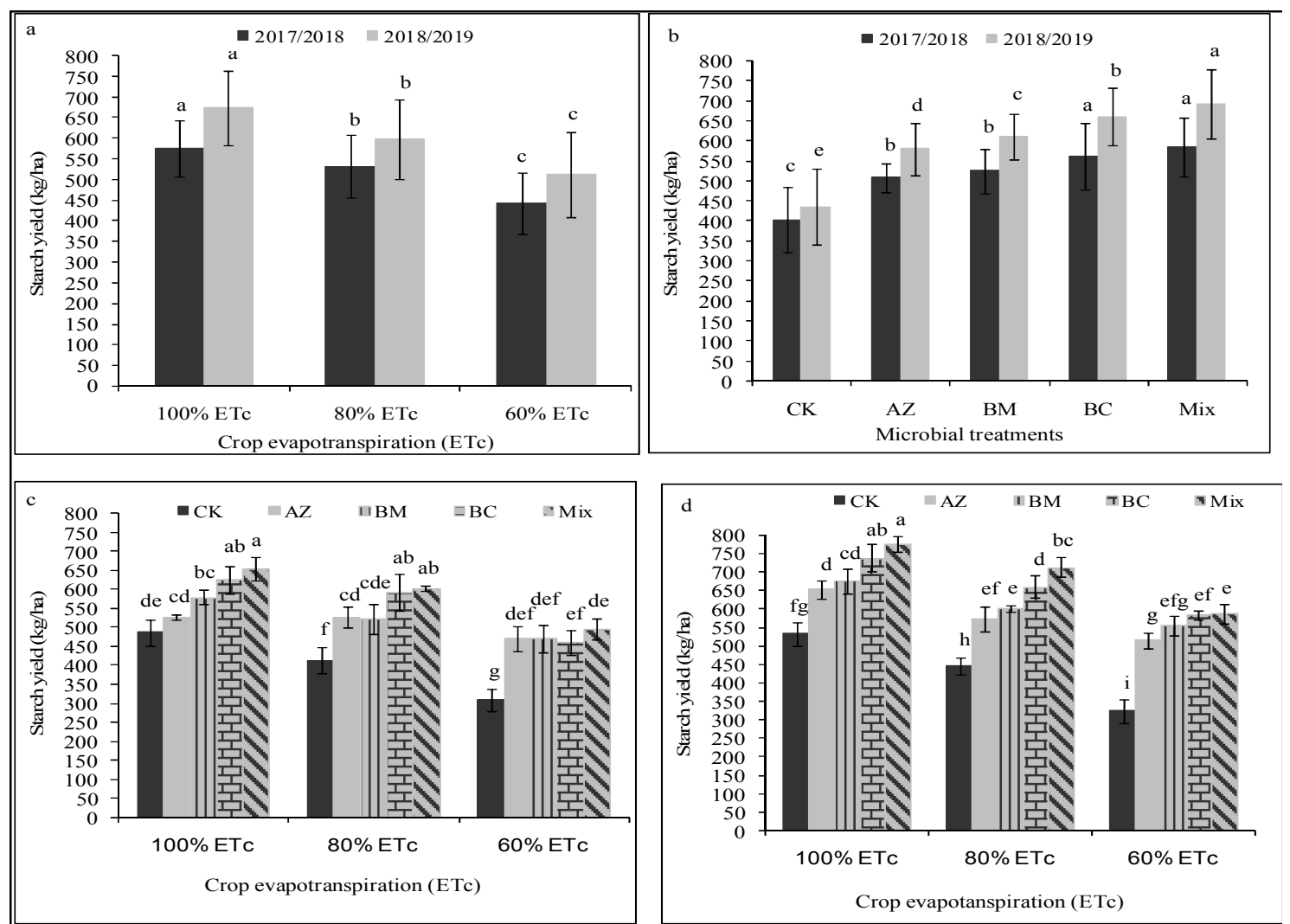

Fig. 4: Influence of irrigation regimes (a), microbial treatments (b) and their interaction during $2017 / 2018$ (c) and 2018/2019 (d) seasons on the starch yield ( $\mathrm{kg} / \mathrm{ha}$ ). Data are presented as means \pm SDs and the different upper letters indicate significant differences at $P<0.05$ level according to Duncan's multiple range tests $(n=15$ in irrigation regimes treatments, $n=9$ in microbial treatments and $\mathrm{n}=3$ in their interaction treatments).

\section{Dry tubers yield (ton/ha)}

Various irrigation regimes and microbial treatments, as well as their interactions, significantly affected the dry tubers yield (ton/ha) at $p<0.01$ during both seasons (Table 5 ). The result of the main factor effect, irrigation regimes, showed that the greater average of dry tubers yield (ton/ha) was obtained when potato plants irrigated with 100\% ETc (5.076 ton/ha and 5.747 ton/ha during 2017/2018 and 2018/2019 seasons, respectively). Meanwhile, among microbial treatments, combined treatment $(\mathrm{AZ}+\mathrm{BM}+\mathrm{BC})$ recorded the highest value of dry tubers yield $\left(5.170\right.$ ton/ha and 5.900 ton/ha during $1^{\text {st }}$ and $2^{\text {nd }}$ seasons, respectively) with increase percentage of 36.28 and 48.09 in both seasons compared to control. This was followed by treated plants with BC (4.918 ton/ha and 5.616 ton/ha). Regarding to the interaction effects, Mix treatment under irrigation regime $100 \% \mathrm{ETc}$ had the greatest value of dry tubers yield during two seasons (5.755 ton/ha and 6.567 ton/ha). Moreover, irrigation potato plants with $80 \%$ ETc and inoculation with combined microbial recorded only $7.04 \%$ and $7.72 \%$ reduction percentage compared to same microbial treatment under $100 \%$ ETc. However, obtained $18.74 \%$ and $27.63 \%$ increase percentage compared to control treatment under $100 \%$ ETc.

\section{Nitrogen uptake (kg/ha)}

The statistical analysis of nitrogen uptake results revealed that there were significant differences among different irrigation regimes, inoculation treatments and their interaction at $p<0.01$ (Table 5). 
Table 5: Dry tubers yield (ton/ha), nitrogen uptake $(\mathrm{kg} / \mathrm{ha})$, phosphorus uptake $(\mathrm{kg} / \mathrm{ha})$ and potassium uptake $(\mathrm{kg} / \mathrm{ha})$ as effected by various irrigation regimes and different microbial treatments as well as their interaction.

\begin{tabular}{|c|c|c|c|c|}
\hline \multirow{2}{*}{ Treat. } & \multicolumn{4}{|c|}{$2017 / 2018$} \\
\hline & $100 \%$ ETc & $80 \%$ ETc & $60 \%$ ETc & Mean \\
\hline & \multicolumn{4}{|c|}{ Dry tubers yield (ton/ha) } \\
\hline CK & $4.476 \pm 0.24^{\mathrm{ef}}$ & $3.960 \pm 0.20^{\mathrm{g}}$ & $2.944 \pm 0.09^{\mathrm{h}}$ & $3.794^{\mathrm{d}}$ \\
\hline $\mathbf{A Z}$ & $4.654 \pm 0.12^{\mathrm{de}}$ & $4.677 \pm 0.10^{\mathrm{de}}$ & $4.233 \pm 0.12^{\mathrm{fg}}$ & $4.521^{\mathrm{c}}$ \\
\hline BM & $5.007 \pm 0.17^{\mathrm{cd}}$ & $4.592 \pm 0.27^{\mathrm{ef}}$ & $4.217 \pm 0.23^{\mathrm{fg}}$ & $4.605^{c}$ \\
\hline BC & $5.488 \pm 0.26^{\mathrm{ab}}$ & $5.211 \pm 0.31^{\mathrm{bc}}$ & $4.055 \pm 0.23^{\mathrm{g}}$ & $4.918^{\mathrm{b}}$ \\
\hline Mix & $5.755 \pm 0.16^{\mathrm{a}}$ & $5.315 \pm 0.12^{\mathrm{bc}}$ & $4.441 \pm 0.03^{\mathrm{ef}}$ & $5.170^{\mathrm{a}}$ \\
\hline \multirow[t]{2}{*}{ Mean } & $5.076^{\mathrm{a}}$ & $4.751^{\mathrm{b}}$ & $3.978^{c}$ & \\
\hline & \multicolumn{4}{|c|}{ Nitrogen uptake (kg/ha) } \\
\hline CK & $57.41 \pm 3.33^{\mathrm{e}}$ & $47.77 \pm 1.81^{\mathrm{fg}}$ & $33.74 \pm 1.88^{\mathrm{h}}$ & $46.31^{\mathrm{e}}$ \\
\hline $\mathbf{A Z}$ & $64.70 \pm 2.23^{\mathrm{c}}$ & $57.76 \pm 0.89^{\mathrm{e}}$ & $49.08 \pm 1.06^{\mathrm{fg}}$ & $57.18^{\mathrm{d}}$ \\
\hline BM & $68.61 \pm 1.78^{\mathrm{b}}$ & $59.55 \pm 1.53^{\mathrm{de}}$ & $50.30 \pm 0.69^{\mathrm{f}}$ & $59.49^{\mathrm{c}}$ \\
\hline BC & $75.54 \pm 2.64^{\mathrm{a}}$ & $62.58 \pm 1.35^{\mathrm{cd}}$ & $46.73 \pm 1.92^{\mathrm{g}}$ & $61.62^{\mathrm{b}}$ \\
\hline Mix & $76.36 \pm 2.09^{\mathrm{a}}$ & $64.66 \pm 2.57^{\mathrm{c}}$ & $50.94 \pm 1.14^{\mathrm{f}}$ & $63.99^{\mathrm{a}}$ \\
\hline \multirow[t]{2}{*}{ Mean } & $68.52^{\mathrm{a}}$ & $58.46^{\mathrm{b}}$ & $46.16^{\mathrm{c}}$ & \\
\hline & \multicolumn{4}{|c|}{ Phosphorus uptake (kg/ha) } \\
\hline CK & $21.10 \pm 1.24^{\mathrm{efg}}$ & $18.23 \pm 0.96^{\mathrm{h}}$ & $13.06 \pm 0.42^{\mathrm{i}}$ & $17.46^{\mathrm{c}}$ \\
\hline $\mathbf{A Z}$ & $22.64 \pm 0.52^{\mathrm{de}}$ & $22.24 \pm 1.85^{\mathrm{de}}$ & $19.43 \pm 1.62^{\mathrm{fgh}}$ & $21.44^{\mathrm{b}}$ \\
\hline BM & $24.48 \pm 1.73^{\text {cd }}$ & $21.52 \pm 1.42^{\mathrm{ef}}$ & $19.45 \pm 0.96^{\mathrm{fgh}}$ & $21.82^{\mathrm{b}}$ \\
\hline BC & $27.09 \pm 1.75^{\mathrm{ab}}$ & $24.67 \pm 1.23^{\mathrm{bcd}}$ & $18.76 \pm 1.39^{\mathrm{gh}}$ & $23.51^{\mathrm{a}}$ \\
\hline Mix & $27.99 \pm 1.51^{\mathrm{a}}$ & $25.22 \pm 1.63^{\mathrm{bc}}$ & $20.50 \pm 1.02^{\text {efgh }}$ & $24.57^{\mathrm{a}}$ \\
\hline \multirow[t]{2}{*}{ Mean } & $24.66^{\mathrm{a}}$ & $22.38^{\mathrm{b}}$ & $18.24^{\mathrm{c}}$ & \\
\hline & \multicolumn{4}{|c|}{ Potassium uptake (kg/ha) } \\
\hline CK & $111.84 \pm 9.06^{\mathrm{de}}$ & $87.53 \pm 4.54^{\mathrm{fg}}$ & $53.32 \pm 4.49^{\mathrm{h}}$ & $84.23^{\mathrm{d}}$ \\
\hline $\mathbf{A Z}$ & $125.89 \pm 10.23^{\mathrm{bc}}$ & $108.24 \pm 7.12^{\mathrm{e}}$ & $76.68 \pm 8.31^{\mathrm{g}}$ & $103.60^{c}$ \\
\hline BM & $127.94 \pm 8.07^{\mathrm{bc}}$ & $113.83 \pm 7.05^{\mathrm{de}}$ & $87.35 \pm 0.58^{\mathrm{fg}}$ & $109.71^{\mathrm{bc}}$ \\
\hline BC & $137.27 \pm 6.70^{\mathrm{ab}}$ & $122.88 \pm 7.47^{\mathrm{cd}}$ & $86.46 \pm 9.35^{\mathrm{fg}}$ & $115.54^{\mathrm{ab}}$ \\
\hline Mix & $143.24 \pm 3.49^{\mathrm{a}}$ & $121.39 \pm 10.07^{\mathrm{cd}}$ & $95.57 \pm 5.08^{\mathrm{f}}$ & $120.07^{\mathrm{a}}$ \\
\hline \multirow[t]{3}{*}{ Mean } & $129.24^{\mathrm{a}}$ & $110.77^{\mathrm{b}}$ & $79.88^{\mathrm{c}}$ & \\
\hline & \multicolumn{4}{|c|}{ 2018/2019 } \\
\hline & \multicolumn{4}{|c|}{ Dry tubers yield (ton/ha) } \\
\hline CK & $4.748 \pm 0.15^{\mathrm{e}}$ & $4.160 \pm 0.12^{\mathrm{g}}$ & $3.043 \pm 0.13^{\mathrm{h}}$ & $3.984^{\mathrm{e}}$ \\
\hline $\mathbf{A Z}$ & $5.509 \pm 0.17^{\mathrm{c}}$ & $4.881 \pm 0.15^{\mathrm{de}}$ & $4.476 \pm 0.12^{\mathrm{f}}$ & $4.956^{\mathrm{d}}$ \\
\hline BM & $5.669 \pm 0.16^{\mathrm{c}}$ & $5.111 \pm 0.14^{\mathrm{d}}$ & $4.807 \pm 0.12^{\mathrm{e}}$ & $5.195^{\mathrm{c}}$ \\
\hline BC & $6.243 \pm 0.20^{\mathrm{b}}$ & $5.612 \pm 0.16^{\mathrm{c}}$ & $4.992 \pm 0.19^{\text {de }}$ & $5.616^{\mathrm{b}}$ \\
\hline Mix & $6.567 \pm 0.19^{\mathrm{a}}$ & $6.060 \pm 0.10^{\mathrm{b}}$ & $5.072 \pm 0.06^{\mathrm{d}}$ & $5.900^{\mathrm{a}}$ \\
\hline \multirow[t]{2}{*}{ Mean } & $5.747^{\mathrm{a}}$ & $5.165^{\mathrm{b}}$ & $4.478^{\mathrm{c}}$ & \\
\hline & \multicolumn{4}{|c|}{ Nitrogen uptake (kg/ha) } \\
\hline CK & $64.11 \pm 1.75^{\mathrm{e}}$ & $53.05 \pm 2.35^{\mathrm{h}}$ & $36.77 \pm 2.19^{\mathrm{i}}$ & $51.31^{\mathrm{e}}$ \\
\hline $\mathbf{A Z}$ & $81.63 \pm 1.56^{\mathrm{b}}$ & $63.53 \pm 2.37^{\mathrm{ef}}$ & $54.60 \pm 2.13^{\mathrm{h}}$ & $66.58^{\mathrm{d}}$ \\
\hline BM & $81.51 \pm 1.09^{\mathrm{b}}$ & $67.79 \pm 2.03^{\mathrm{d}}$ & $59.50 \pm 1.23^{\mathrm{g}}$ & $69.60^{c}$ \\
\hline BC & $90.01 \pm 1.74^{\mathrm{a}}$ & $72.13 \pm 1.42^{\mathrm{c}}$ & $60.24 \pm 2.00^{\mathrm{fg}}$ & $74.13^{\mathrm{b}}$ \\
\hline Mix & $91.54 \pm 2.81^{\mathrm{a}}$ & $78.73 \pm 2.18^{\mathrm{b}}$ & $61.55 \pm 2.65^{\mathrm{efg}}$ & $77.27^{\mathrm{a}}$ \\
\hline \multirow[t]{2}{*}{ Mean } & $81.76^{\mathrm{a}}$ & $67.05^{\mathrm{b}}$ & $54.53^{\mathrm{c}}$ & \\
\hline & \multicolumn{4}{|c|}{ Phosphorus uptake $(\mathrm{kg} / \mathrm{ha})$} \\
\hline CK & $21.16 \pm 0.93^{\mathrm{de}}$ & $18.17 \pm 0.77^{\mathrm{f}}$ & $12.77 \pm 0.51^{\mathrm{g}}$ & $17.37^{\mathrm{d}}$ \\
\hline $\mathbf{A Z}$ & $25.41 \pm 0.67^{\mathrm{c}}$ & $21.95 \pm 1.27^{\mathrm{d}}$ & $19.44 \pm 1.32^{\mathrm{ef}}$ & $22.27^{\mathrm{c}}$ \\
\hline BM & $26.28 \pm 1.01^{\mathrm{c}}$ & $22.85 \pm 1.03^{\mathrm{d}}$ & $21.03 \pm 1.18^{\text {de }}$ & $23.39^{\mathrm{c}}$ \\
\hline BC & $29.04 \pm 1.17^{\mathrm{ab}}$ & $25.22 \pm 2.20^{\mathrm{c}}$ & $21.82 \pm 0.81^{\mathrm{d}}$ & $25.36^{\mathrm{b}}$ \\
\hline Mix & $30.34 \pm 1.21^{\mathrm{a}}$ & $27.29 \pm 1.22^{\mathrm{bc}}$ & $22.17 \pm 1.25^{\mathrm{d}}$ & $26.60^{\mathrm{a}}$ \\
\hline \multirow[t]{2}{*}{ Mean } & $26.44^{\mathrm{a}}$ & $23.09^{\mathrm{b}}$ & $19.45^{\mathrm{c}}$ & \\
\hline & & Potass & kg/ha) & \\
\hline CK & $113.44 \pm 7.78^{\mathrm{fg}}$ & $87.80 \pm 5.28^{i j}$ & $53.38 \pm 4.64^{\mathrm{k}}$ & $84.87^{\mathrm{d}}$ \\
\hline $\mathbf{A Z}$ & $141.44 \pm 7.67^{\mathrm{bc}}$ & $108.09 \pm 3.86^{\mathrm{g}}$ & $78.50 \pm 5.91^{\mathrm{j}}$ & $109.34^{\mathrm{c}}$ \\
\hline BM & $139.51 \pm 7.12^{\mathrm{c}}$ & $119.54 \pm 4.13^{\text {ef }}$ & $95.17 \pm 3.97^{\mathrm{hi}}$ & $118.07^{\mathrm{b}}$ \\
\hline BC & $151.20 \pm 6.34^{\mathrm{ab}}$ & $127.96 \pm 9.09^{\mathrm{de}}$ & $102.67 \pm 6.31^{\mathrm{gh}}$ & $127.28^{\mathrm{a}}$ \\
\hline Mix & $157.54 \pm 4.86^{\mathrm{a}}$ & $133.46 \pm 7.66^{\mathrm{cd}}$ & $104.61 \pm 6.03^{\mathrm{gh}}$ & $131.87^{\mathrm{a}}$ \\
\hline Mean & $140.62^{\mathrm{a}}$ & $115.37^{\mathrm{b}}$ & $86.86^{\mathrm{c}}$ & \\
\hline
\end{tabular}

Values are given as the mean \pm standard deviation. Different letters indicate statistically differences according to Duncan's multiple range tests.

CK: Control, AZ: Azotobacter chroococcum + Azospirillum brasilense, BM: Bacillus megaterium, BC: Bacillus circulans Mix: Combined $\mathrm{AZ}+\mathrm{BM}+\mathrm{BC})$. 
Compared to other irrigation regimes, $100 \%$ ETc showed the highest nitrogen uptake in both seasons $(68.52 \mathrm{~kg} / \mathrm{ha}$ and $81.76 \mathrm{~kg} / \mathrm{ha})$. In generally, inoculation treatments compared to control increased nitrogen uptake highly significant. Mix treatment enhanced nitrogen uptake by $38.18 \%$ and $50.59 \%$ compared to control. The combination of $100 \% \mathrm{ETc}$ and Mix treatment induced greatest nitrogen uptake in both seasons $(76.36 \mathrm{~kg} / \mathrm{ha}$ and $91.54 \mathrm{~kg} / \mathrm{ha})$. Additionally, same inoculation treatment (AZ+BM+BC) under irrigation regime $80 \%$ ETc obtained reduction percentage of $15.32 \%$ and $13.99 \%$ compared to irrigation regimes $100 \%$ ETc. However, increased nitrogen uptake by $12.63 \%$ and $22.80 \%$ in the $2017 / 2018$ and 2018/2019 seasons, respectively, compared to control under irrigation regime $100 \%$ ETc.

\section{Phosphorus uptake (kg/ha)}

The statistical analysis of phosphorus uptake results reported that there were significant differences among various irrigation regimes and microbial treatments at $p<0.01$ (Table 5).Plants irrigated with $100 \%$ ETc had the highest phosphorus accumulation compared with other irrigation regimes, which recorded $24.66 \mathrm{~kg} / \mathrm{ha}$ and $26.44 \mathrm{~kg} / \mathrm{ha}$ in 2017/2018 and 2018/2019 seasons, respectively (Table 5). Moreover, the results of the microbial treatment effect indicated that plants treated with Mix microbial treatment accumulated highest phosphorus amounts during both seasons $(24.57 \mathrm{~kg} / \mathrm{ha}$ and $26.60 \mathrm{~kg} / \mathrm{ha}$ ) with $40.72 \%$ and 53.14 increase percentage compared to un-inoculated treatment (CK). The results of interaction effect showed that potato plants under irrigation regime $100 \%$ ETc and treated with combined microbial accumulated more phosphorus than other treatments (27.99 $\mathrm{kg} / \mathrm{ha}$ and $30.34 \mathrm{~kg} / \mathrm{ha}$ during first and second seasons, respectively). Phosphorus accumulation amount under irrigation regime $80 \%$ ETc and Mix treatment recorded $19.53 \%$ and $28.97 \%$ increase percentage compared to control treatment under irrigation regime $100 \%$ ETc.

\section{Potassium uptake (kg/ha)}

The statistical analysis of potassium uptake results conducted that there were significant differences among different irrigation regimes and bacterial treatments at $p<0.01$ (Table 5). The total amount of potassium accumulation during 2017/2018 and 2018/2019 seasons was higher under irrigation regime $100 \%$ ETc than $80 \%$ ETc and $60 \%$ ETc, which were $129.24 \mathrm{~kg} / \mathrm{ha}$ and $140.62 \mathrm{~kg} / \mathrm{ha}$, respectively (Table 5). This was followed by irrigation regime $80 \%$ ETc $(110.77 \mathrm{~kg} / \mathrm{ha}$ and $115.37 \mathrm{~kg} / \mathrm{ha}$ during $1^{\text {st }}$ and $2^{\text {nd }}$ seasons, respectively. Over different irrigation regimes, microbial inoculation enhanced potassium accumulation compared to control treatment. Mix treatment recorded highest potassium quantity $(120.07 \mathrm{~kg} / \mathrm{ha}$ and $131.87 \mathrm{~kg} / \mathrm{ha})$, with no significant difference with $\mathrm{BC}$ treatment in both seasons $(115.54 \mathrm{~kg} / \mathrm{ha}$ and $127.28 \mathrm{~kg} / \mathrm{ha})$. According to the interaction effects between various irrigation regimes and different microbial treatments, Mix treatment under irrigation regime $100 \%$ ETc had the greatest value of potassium accumulated amount during both seasons $(143.24 \mathrm{~kg} / \mathrm{ha}$ and 157.54 $\mathrm{kg} / \mathrm{ha}$ ), with no statistically significant differ with $\mathrm{BC}$ under the same irrigation regime. Furthermore, treated potato plants under irrigation regime $80 \%$ ETc with combined microbial give $121.39 \mathrm{~kg} / \mathrm{ha}$ and $133.46 \mathrm{~kg} / \mathrm{ha}$ potassium amount, with $8.54 \%$ and $17.65 \%$ increase percentage compared to control under irrigation regime $100 \%$ ETc. This was not significant differ than BC under irrigation regime $80 \%$ ETc during both seasons.

\section{Discussion}

There are often different responses among crops to water deficit or drought stress. Potato considered as drought sensitive crop and the decrements of its productivity are related with decreasing required water amount during different growth stages. Previous reports concluded that water deficit cause yield reduction by decreasing the growth of crop canopy and biomass (Fabeiro et al., 2001; Yuan et al., 2003; Onder et al., 2005; Kaur et al., 2005). The variations in irrigation water applied among different growth stages might be due to the loss of moisture were mostly by soil surface evaporation at the beginning of the growth stage because potato canopy has not established yet. However, a gradual increase in water consumption was recorded due to plant develop and the increase in irrigation water applied under $100 \%$ ETc could be attributed to the increase in direct evaporation. Therefore, the seasonal irrigation water applied is higher under $100 \%$ ETc followed by 80 and $60 \%$ ETc for potato during the two growth seasons. These results are in the same trend with those reported by Abdalla et al. (1990); El-Koliey et al. (2001); Eid et al. (2013), Ati et al. (2013) and Badawy et al. (2019). 
Potato yield was significantly increased by inoculated potato plants with various bacteria compared to control treatment under various irrigation regimes. Several studies indicated the positive effects of bio-fertilizer on growth of plant and increase the crop yield significantly compare to noninoculated plants (Hammad and Abdel-Ati 1998; Atimanav and Adholeya 2002; Clarson 2004; Malboobi et al. 2009; Abou El-Khair and Nawar 2010; Abou-Zeid Bakry 2011; El-Sayed et al. 2015). The obtained results are in accordance with Zaghloul (2002) who found that the growth parameters were significantly increased potato tuber inoculated by B. megaterium. In the same trend, Azotobacter spp. and Azospirillum spp., by producing some phytohormones such as auxin, cytokinin, gibberellins and abscisic acid, can promote plant growth and enhance plant productivity (Bottini et al. 2004). Moreover, Azotobacter spp. could enhance plant growth and production because it can help the plant to fight against many plant pathogens for the reason that it can also produce antifungal compounds (JenHshuan 2006). The positive effects due to use microbial inoculation might be attributed to enhance the efficiency of water and nutrients absorption (Dalla Santa et al. 2004; Mohammadi et al. 2013). Bottini et al. (2004) mentioned that Azospirillum spp. inoculation enhance plant growth through increase the number of lateral roots and root hairs results in increase the root surface, which results in enhance nutrient uptake and improve water status. Lin (1991) indicated that treated soil with microorganisms improve soil structure and become less compact, more friable and better drained, and this could lead to provided better growing conditions for potato growth and tuber production. Previous studies reported that the positive effect of microorganism inoculation on yield and yield component could be due to enhance root growth and functions, increasing mineral uptake into the plant, phytohormones production i.e. IAA, GAs and cytokinin, also cause reduction of abscic acid (Stancheva et al. 1995; Elhakim et al. 2016). In addition, Kawther et al. (2002) indicated that phytohormones play important role in plant growth stimulation. Also, El-Sayed et al. (2015) reported that using organic and bio-fertilizer increased significantly the marketable tubers. Production of plant growth regulators by microorganism could increase the positive effect of bio-fertilizers in increasing total and marketable potato yield (Norman et al., 2003).

The trends of irrigation water use efficiency are negatively correlated with the total amount of irrigation water, total tuber yield and dry biomass of potato plants (Yuan et al. 2003; Abou El-Khair et al., 2011). The obtained trends of IWUE are in general agreement with those reported by Al-Aubiady (2005); Mahmoud and hafez (2010); Abd-All et al. (2017); Khan et al. (2017) and Badawy et al. (2019). Furthermore, in melon, IWUE did not increased by increasing irrigation levels as reported by Al-Mefleh et al. (2012) and Yaseen et al. (2014); and lower irrigation treatments induce higher values of IWUE (Abd El-Mageed and Semida 2015). This was confirmed by Ertek et al. (2004) who concluded that the highest values of IWUE for summer squash were obtained under the lowest irrigation conditions. In the same direction, El-Gindy et al. (2009) showed that lower water amounts ( $60 \%$ of ETc) recorded higher IWUE than drip-irrigated summer squash with higher water amounts $(80 \% \mathrm{ETc})$. In contrast, Cantore et al. (2014) reported that IWUE was not influenced by the applied supplementary irrigation, which the IWUE values were 10.7 and 10.6 for full irrigation and $50 \%$ irrigation.

The high tuber quality could be comrade to height absorbance and effective use of nutrients by potato plants for proteins synthesis and carbohydrates metabolism, which are answerable to dry matter accumulation (Jatav et al., 2013). The obtained results of protein and starch yield, dry tubers yield, as well as nitrogen, phosphorus and potassium uptake, were influenced negatively by water deficit and increased significantly when potato plants inoculated by bacteria compared to control. The increases in the protein yield, starch yield, dry tubers yield might be attributed to the high content of leaves from the nitrogen, phosphorous and potassium elements that donate efficiently to the protein and starch composition as well as increase in total dissolved solids in the potato tubers (El-Zehery, 2019). Nitrogen is directly included in the synthesis of amino acids, which are the essential compounds for protein synthesis (Barak, 1999). Phosphorous is enters inside the synthesis of DNA and RNA, which directly influences protein synthesis and amelioration of crop yield and quality (Scalenghe et al., 2012). In addition, potassium effects the irrigation economy and plant growth through its effects on water absorbance, root development, upkeep of turgor, stomatal regulation, transpiration, enzyme activation and carbohydrate metabolism, as well as potassium enhancement the efficiency of nitrogen uptake and consequently increases in protein synthesis. (Mfilinge et al. 2014; Elhakim et al. 2016).

The decrements of $\mathrm{N}$ and $\mathrm{P}$ under water deficit conditions are related with decreasing of water potential in rhizosphere and cells of the plant (Mamnabi et al. 2020). In addition, Bista et al. (2018) 
stated that drought decreases the concentration of nitrogen and phosphorous in plants consequently decreasing productivity. On the other side different biochemical and physiological processes are altered by water deficit, such as photosynthesis (Pagter et al., 2005) and the metabolism of amino acids, protein, carbohydrates and other organic compounds (Šircelj et al., 2005). In this respect, Abou El-Khair et al. (2011) declared that irrigation by water quantity of 2976 or $4167 \mathrm{~m}^{3} /$ ha recorded the highest values of carbohydrates, starch, dry matter, $\mathrm{N}$ and $\mathrm{P}$ content in tuber tissues as compared with the lowest irrigation water quantity $\left(1786 \mathrm{~m}^{3} / \mathrm{ha}\right)$.

The increases in N, P and K uptake by potato tubers inoculated by Azotobacter chroococcum + Azospirillum brasilense attributed to the higher absorption of available NPK, that's might be were abundantly usable in soil. In addition, species of Azotobacter and Azospirillum, these are free-living nitrogen fixing bacteria are obligate aerobes that fix atmospheric nitrogen and transferring them to plant as usable forms in the soil, thereby converting them available to plants without symbiosis. Furthermore, it has an ability to fix nitrogen between $20-40 \mathrm{~kg} / \mathrm{ha}$ and save $\mathrm{N}$-fertilizer in some cereals, vegetable and legume crops such as maize, sorghum, sugarcane, potato, onion and pigeonpea (Bhattacharjee and Dey, 2014; Jehangir, et al. 2017). These quantity of fixed nitrogen was important, especially in these current investigation with calcareous soils contained $91.1 \%$ sand, only $0.01 \%$ total nitrogen (measured by micro-kjeldahl procedure) and high $\mathrm{pH}$ value 8.44 (measured by a glass electrode in 1:1 soil water suspension). On the other hand, potato crop grown in arid region in these currant investigation was fertilized by modest $\mathrm{N}$-fertilizer level (only, $214.3 \mathrm{~kg} / \mathrm{ha}$ ). On the other side, $\mathrm{N}$-fixer bacteria can produced growth-regulating materials and enhance crop yield by $15-30 \%$ (Singh and Singh, 2011). Moreover, these $\mathrm{N}$-fixing bacterial species can significantly increment other nutrients available to the plant (Mohammadi et al. 2013). In this respect, Mahendran et al. (1996) showed that inoculation with Azospirillum sp and B. megaterium increased potato tubers dry matter contents and NPK uptake.

Usually soil $\mathrm{pH}$ is the most crucial chemical characteristic influencing nutrient precipitation, solubility, availability and sorption (Basta et al. 2005). Optimum availability to plants of both applied and natural phosphorus as well as nitrogen and potassium is in the $\mathrm{pH}$ ambit of 6.0 to 7.5 . At higher $\mathrm{pH}$ values, especially in calcareous soils calcium and magnesium cations react with phosphate anions to form insoluble phosphate compounds that cannot be absorbed by crops (Wahba et al. 2019). Taking into consideration that, the current investigation soil was sandy calcareous soils, with $29.80 \%$ total $\mathrm{CaCO}_{3}$ (measured by a Schreiber calcimeter) and $\mathrm{pH}$ was 8.44 .Thus, this physico-chemical properties, was reflected on available phosphorus (only $7.01 \mathrm{ppm}$ of available phosphorus, measured coloremetrically. by the stannous chloride phosphomolybdic-sulfuric acid method as dscrepted by Jackson, (1973). On the other hand, moves of phosphorus in calcareous soils is very little from the point of application. Consequently, P availability and uptake by plants will be limited (Rubiez et al. 1991). On the other side, potassium in the soil emerges mostly as silicate minerals, which are unavailable to plants. These minerals are created available only when they are solubilized or slowly weathered (Ju et al., 2018). The additions in $\mathrm{P}, \mathrm{K}$ and $\mathrm{N}$ uptake by potato tubers inoculated by Bacillus megaterium and/or Bacillus circulans attributed to the higher absorption of available $\mathrm{P}, \mathrm{K}$ and $\mathrm{N}$, that's might be were abundantly available in soil.

Phosphate solubilizing bacteria (PSB) can solubilize the fixed phosphorus in calcareous soil and other soils. PSB have the capability to transfer chemical unavailable $\mathrm{P}$ form to soluble forms $\mathrm{H}_{2} \mathrm{PO}^{-}$ and $\mathrm{HPO}_{4}^{-2}$, through the process of enzymes, organic and inorganic acids production (low molecular weight organic acids, usually ketogluconic and gluconic acids). Carboxy and hydroxy groups of acids chelate cations $(\mathrm{Ca}, \mathrm{Mg}, \mathrm{Al}$ and $\mathrm{Fe})$ and reduced the $\mathrm{pH}$ of the soil and create the dissolution of fettered forms of phosphate producing them available to plants as well as other nutrients will be more available (Han et al. 2006; Yang et al. 2009; Mohammadi and Sohrabi 2012; Ju et al. 2018). In addition, bacteria are more efficient in P solubilization than fungi (Mohammadi and Sohrabi, 2012). Bacillus megatherium and Bacillus circulans could be commit as the most important strains (Mohammadi and Sohrabi, 2012). Ju et al. 2018 reported that some phosphate solubilizing microbial like Bacillus species has been found to solubilize phosphorus and potassium as well as mobilize phosphorus and potassium. Thus, more phosphorus and potassium uptake is prospective especially with potato shallow rooting system (Stark et al., 2004). Opena and Porter, (1999) mentioned that around 85\% of potato root is intensive in the upper $30 \mathrm{~cm}$ soil layer. In this respect, Abd El-Daym et al. (2019) conducted that combined application of different sources of nitrogen fertilizers (organic and inorganic N) with biofertilizer significantly enhanced contents of protein, starch, dry matter, nitrogen, phosphorus, 
potassium and calcium in potato tubers as well as increased soil nutrient contents, such as usable $\mathrm{N}$ $\left(\mathrm{NH}_{4}-\mathrm{N}\right.$ and $\left.\mathrm{NO}_{3}-\mathrm{N}\right)$, usable $\mathrm{P}$ and exchangeable $\mathrm{K}$ content. Furthermore El-Zehery, (2019) suggested that organic fertilizer + biofertilizer (Bacillus, Azospirillum, Azotobacter and Pseudomonas spp) guide to a significant increment in protein, starch, dry matter, nitrogen, phosphorus and potassium content in potato tubers as compared with control (without organic fertilizer or biofertilizer).

Potassium solubilizing bacteria like as Bacillus Spp solubilize silicates by making organic acids, which motive the decomposition of silicates and assist in the transit of metal ions thereby making them usable to plants (Ju et al., 2018). Furthermore, increase in potassium uptake might be attributed to the role of microorganisms in providing great amounts of water-soluble and amorphous potassium which was reflected in plant uptake (Afify and Bayoumy, 2001). Thus, Potassium solubilizing microbial are wide uses as bio-fertilizers. In this respect, Elhakim et al. (2016) hypothesis that, application of $\mathrm{K}$ fertilizer at $286 \mathrm{~kg} \mathrm{~K}_{2} \mathrm{O} / \mathrm{ha}$ with application of potassium biofertilizer Bacillius Circulans gave the highest mean value of potassium content in leaves, starch and potassium content in potato tubers. In addition, Elkholy et al. (2012) observed that inoculation with Bacillus circulans as silicate dissolving bacteria with different potassium sources increased tuber content of protein and carbohydrate as well as plant shoot, plant tubers and soil usable content of $\mathrm{N}, \mathrm{P}, \mathrm{K}, \mathrm{Fe}, \mathrm{Mn}, \mathrm{Zn}$ and $\mathrm{Cu}$ compared to the control (without K sources).

Generally, use of bacteria could increase the nutrient uptake through some modification of rhizosphere physic-chemical properties such as enhancing soil cation exchange capacity and some other biochemical responses in root tissues (Adhikary, 2012). Improve of root nutrient absorption under various environmental conditions i.e. water stress might be because the reason of PGPRs treatments increased the photo assimilate translocation to the root tissues. PGPRs could be increase the activity of some important enzymes involved in nitrogen metabolizing like nitrate reductase (NR), thereby improving the $\mathrm{N}$ content under water deficit (Ansari and Ahmad, 2019).

In respect to the obtained results in the current investigation, the extend in yield and yield compounds, dry tubers yield, protein and starch yield, and NPK uptake increases in the inoculation treatments compared to control treatments under different irrigation regimes might be attributed to the beneficial effects of microorganisms and their important roles in supplying plants with nutrients, plant growth regulators and production of some phytohormones and antifungal. Consequently, use of microbial inoculation could be effective method to alleviate the impact of water stress on plant growth and crop production.

\section{Conclusion}

Increasing water deficit induced decrease in all studied parameters exhibit irrigation water use efficiency and economic productivity of irrigation water. However, with application of microbial inoculation potato growth, total and marketable yield, dry tubers yield, protein and starch yield, and uptake of N, P, and $\mathrm{K}$ were enhanced under various irrigation regimes compared to control. Especially the combined microbial treatment $(\mathrm{AZ}+\mathrm{BM}+\mathrm{BC})$ increased significantly the above mentioned characters.

\section{References}

Abd- All, A. E., A.E. El-Namas and E.M. EL-Naggar, 2017. Effect of Humic Acid and Foliar Application of Different Potassium Sources on Yield, Quality and Water Use Efficiency of Sweet Potato Grown under Drip Irrigation in Sandy Soil, Alexandria Science Exchange Journal,. 38(3): 543-553.

Abd El-Daym, E.A., M.B.I. El-Sawy and M.A. El-Helaly, 2019. Combined application of different sources of nitrogen fertilizers for improvement of potato yield and quality. Plant Archives, 19 (2): 2513-2521.

Abd El-Mageed, T.A. and W.M. Semida, 2015. Effect of deficit irrigation and growing seasons on plant water status, fruit yield and water use efficiency of squash under saline soil. Scientia Horticulturae 186: 89-100. https://doi.org/10.1016/j.scienta.2015.02.013.

Abdalla, I.M., Shafshak, S. Nadia, F.A. Abo-Sedera and L.A. Abd El-Rahman, 1990. Effect of water regime and level on NPK fertilization on carrot I- vegetative growth and its chemical composition. Annals of Agric. Sci., Moshtohor Univ., 28: 2517-2528. 
Abou El-Khair, E.E. and D.A.S. Nawar, 2010. Effect of phosphorus and some biostimulants on growth yield phosphorus use efficiency and tuber quality of potato plants growth in sandy soil. Zagazig J. of Agric. Res., 37(5):1077-1103.

Abou El-Khair, E.E., D.A.S. Nawar and H.M.E. Ismail, 2011. Effect of irrigation water quantity and farmyard manure on potato plant grown in sandy soil. Egypt. J. Agric. Res. 89(1): 317-334.

Abou-Hussien, E.A., A.M. Elbaalawy and M.M. Hamad, 2019. Chemical properties of compost in relation to calcareous soil properties and its productivity of wheat. Egypt. J. Soil. Sci. 59(1): 8597.

Abou-Zeid, M.Y. and M.A.A. Bakry, 2011. Integrated effect of bio-organic manures and mineral fertilizers on potato productivity and the fertility status of a calcareous soil. Austr. J. Basic \& Applied Sci., 5(8): 1385-1399.

Adhikary, S., 2012. Vermicompost, the story of organic gold: a review. Agric. Sci. 37: 905-917.

Afify, A.H. and S.M.M. Bayoumy, 2001. Effect of certain silicate bacteria on primary silicate minerals. Egypt J. Agric Sci., Mansoura Univ., 26(5): 3111-3125.

Al-Aubiady, A.M.S., 2005. Physiological Studied to Improve Growth, Yield, Tuber Seed Production and Decrease the Water Stress in Potato (Solanum tuberosum L.) Ph.D Thesis - College of Agric. and Forestry - Mosul Univ. Iraq.

Allen, G. R., L.S. Pereira, D. Raes and M. Smith, 1998. Crop evapotranspiration, guidelines for competing crop water requirements. FAO. Irrigation and drainages paper 56. Rome, Italy.

Al-Mefleh, N.K., N. Samarah, S. Zaitoun and A. Al-Ghzawi, 2012. Effect of irrigation levels on fruit characteristics, total fruit yield and water use efficiency of melon under drip irrigation system. J. Food Agric. Environ. 2: 540-545.

Amiour, N., S. Imbaud, G. Clément, N. Agier, M. Zivy, B. Valot, T. Balliau, P. Armengaud, I. Quilleré, R. Cañas, T. Tercet-Laforgue and B. Hirel, (2012) The use of metabolomics integrated with transcriptomic and proteomic studies for identifying key steps involved in the control of nitrogen metabolism in crops such as maize. Journal of Experimental Botany 63(14), 5017-5033.DOI: https://doi:10.1093/jxb/ers186.

Ansari, F.A., I. Ahmad, 2019. Alleviating drought stress of crops. Through PGPR: mechanism. Microbial interventions in agriculture and environment: 2: rhizosphere, microbiome and agroecology, 341-358.

Ati, A.S., F. Al-Sahaf, D.H. Wally and T.E. Thamer, 2013. Effects of Potassium Humate Fertilizers and Irrigation Rate on Potato Yield and Consumptive Use under Drip Irrigation Method, Journal of Agricultural Science and Technology A 3: 803-810

Atimanav, G. and A. Adholeya, 2002. AM inoculation of five tropical fodder crops and inoculum production in unfertilized soil amended with organic matter. Biol Fert Soil 35:214-218.

Azab, E., 2016. Effect of water stress and biological fertilization on maize growth, chemical composition and productivity in calcareous. Am. J. Plant Physiol. 11: 1-11.

Badawy, A.S., Kh.M. Abd El- Latif, M.M. Hefzy; and A.M. Attia, 2019. Effect of yeast extract and humic acid on productivity and quality of potato (Solanum tuberosum) under different regimes of drip irrigation system in newly reclaimed soils. Fayoum J. Agric. Res. \& Dev., 33(1):102-113.

Barak, 1999. Essential elements for plant's growth published by Nature publishers. 1- 5 .

Baranowska, A.J., 2018. Yield of dry matter and starch of edible potato tubers in conditions of application of growth biostimulators and herbicide. Acta Agroph., 25(4), 397-407. https://doi: $10.31545 /$ aagr/99073

Basta, N.T., J.A. Ryan and R.L. Chaney, 2005.Trace element chemistry in residual-treated soil: key concepts and metal bioavailability. J. Environ. Qual., 34:49-63.

Bhattacharjee, R. and U. Dey, 2014. Biofertilizer, a way towards organic agriculture: a review. African Journals of Microbiology Research, 8 (24):2332-2342. https://doi: 10.5897/AJMR2013.6374

Bhattacharyya, P.N. and D.K. Jha, 2012. Plant growth-promoting rhizobacteria (PGPR): emergence in agriculture. World Journal of Microbiotechnology. 28(4):1327-50.

Bista, D. R., S.A. Heckathorn, D.M. Jayawardena, S. Mishra and J.K. Boldt, 2018. Effects of drought on nutrient uptake and the levels of nutrient uptake proteins in roots of drought-sensitive and tolerant grasses. Plants Journal, (7):28:1-16. https://doi:10.3390/plants7020028.

Bottini, R., F. Cassan and P. Piccoli, 2004. Gibberellin production by bacteria and its involvement in plant growth promotion and yield increase. Appl Microbiol Biotechnol 65: 497-503. 
Brody, N.C. and R.R. Weil, 1999. The Nature and Properties of Soils. $12^{\text {th }}$ Edition. Prentice Hall, Upper Saddle River, New Jersey.

Burton, W.G., 1948. The Potato. Chapman and Hall, London. Pp.319.

Cantore, V., F. Wassar, S.S. Yamaç, M.H. Sellami, R. Albrizio, A.M. Stellacci and M. Todorovic, 2014. Yield and water use e ciency of early potato grown under di_erent irrigation regimes. Int. J. Plant Prod., 8: 409-428.

Chaves, M.M., J.P. Maroco and J.S. Pereira, 2003. Understanding plant responses to drought-from genes to the whole plant. Funct. Plant Biol. 30: 239-264. doi:10.1071/FP02076.

Clarson, D., 2004. Potash biofertilizer for ecofriendly agriculture. Agro-clinic and Research Centre, Poovanthuruthu, Kottayam (Kerala), India, 98-110.

Dalla Santa, O.R., R.F. Hernandez, G.L. Michelena Alvarez, P.R. Junior and C.R. Soccol, 2004. Azospirillum sp. inoculation in wheat, Barley and oats seeds greenhouse experiments. Braz. Arch. Biol. Technol. 47(6):843-850.

Delshadi, S., M. Ebrahimi and E. Shirmohammadi, 2017. Effectiveness of plant growth promoting rhizobacteria on Bromus tomentellus Boiss seed germination, growth and nutrients uptake under drought stress. South African Journal of Botany, 133: 11-18. https://doi.org/10.1016/j.sajb.2017.07.006.

Dupuis, J.H. and L. Qiang, 2019. Potato starch: a review of physicochemical, functional and nutritional properties. American Journal of Potato Research. https://doi.org/10.1007/s12230-018-09696-2

Eid, T.A., S.M.M. Ali and N.H. Abou-Baker, 2013. Influence of Soil Moisture Depletion on Water Requirements, Yield and Mineral Contents of Potato, Journal of Applied Sciences Research, 9(3): 1457-1466.

Ekin, Z., 2011. Some analytical quality characteristics for evaluating the utilization and consumption of potato (Solanum tuberosum L.) tubers. African Journal of Biotechnology, 10(32): 6001-6010. https://doi: 10.5897/AJB11.042

El-Damaty, A.H., A. El-Leboudi and A. Robishy, 1973. Some studies on the calcareous soils of Egypt. In: FAO Soils Bulletin 21 - Calcareous Soils, pp 233 to 237 .Food and Agriculture Organization of the United Nations, Rome.

El-Gindy, A.G.M., S. El-Banna, M.A. El-Adl and M.F. Metwally, 2009. Effect of fertiliza-tion and irrigation water levels on summer squash yield under drip irrigation. Misr J. Agric. Eng., 26: 94 106.

Elhakim, S.A.Z., D.S. El-Mesirry and M.M. Yousry 2016. Impact of potassium fertilization rates and Bacillus circulans on the growth, yield and color of processed potato (Solanum tuberosum L.) tubers chips. Alexandria Science Exchange Journal, 37(4): 594-605.

Elkholy, M.M., S.A. El-Tohamy and W.A. Hafez, 2012. Retionalization of mineral potassium fertilizer by using biodesolving potassium and its effect on yield and quality potatoes. J. Soil Sci. and Agric. Eng., Mansoura Univ., 3 (12): 1275- 1285.

El-Koliey, M.M., S.E. Soliman and H.M. Eid, 2001. Estimation of crop water needs in Assiut Governorate. 6th conference-meteorology \& sustainable development. April 2- 4, Cairo, Egypt.

El-Sayed, S.F., H.A. Hassan and M.M. El-Mogy, 2015. Impact of Bio- and Organic Fertilizers on Potato Yield, Quality and Tuber Weight Loss after Harvest. Potato Res. 58: 67-81. https://doi.org/10.1007/s11540-014-9272-2.

El-Zehery, T.M., 2019. Incorporated use impact of organic, bio and mineral fertilizers on potato (Solanum tuberosum. L) productivity and quality. J. of Soil Sciences and Agricultural Engineering, Mansoura Univ., 10 (12): 857-865. https://doi: 10.21608/jssae.2019.79681.

Ertek, A., S. Sensoy, I. Gedik and C. Kücükyumuk, 2006. Irrigation scheduling based on pan evaporation values for cucumber (Cucumis sativus L.) grown under field conditions. Agric. Water Manage, 81: 159-172.

Fabeiro, C.M.D.S.O.F. and J.A. de Juan, 2001. Yield and size of deficit irrigated potatoes. Agricultural Water Management, 48: 255-266.

FAO, 1979. Yield response to water. Irrigation and drainage paper 33, Rome, Italy.

Farag, Jr.M.I., M.A. Abd-Alla, M.F. Mohamed and M.H. Aboul-Nasr, 2013. Effect of biofertilization on yield and quality of some potato cultivars (Solanum tuberosum L.).International Journal of Agriculture and Food Science Technology, 4(7): 695-702. 
Fife, D.N and E.K.S. Nambiar, 1997. Changes in the canopy and growth of Pinusradiata in response to nitrogen supply. For.Ecol. Manage., 93: 137e152.

Freed, R.D., S.P. Eisensmith, S. Goelz, D. Reicozky, W.W. Smail and P. Woberg, 1987. MSTAT-C 2.1. A software package program for the design, management and analysis of agronomic research experiments. Crop and Soil Sciences Department; Michigan State University, USA.

Goyal, S.S., R. Tischner and A.S. Basra, 2005. Enhancing the efficiency of nitrogen utilization in plants. Haworth: Haworth Press, Inc.489.

Halvorson, A.D. and C.A. Reule, 1994. Nitrogen fertilizer requirements in an annual dryland cropping system. Agron. J. 86: 315e318.

Hammad, A.M.M. and Y.Y. Abdel-Ati, 1998. Reducing of nitrate content of potato tuber via biofertilization with Azospirillum and via mycorrhizal fungi. J. Agric. Sci. Mansoura Univ., 23:2597-2610.

Han, H.S., Supanjani and K.D. Lee, 2006. Effect of co-inoculation with phosphate and potassium solubilizing bacteria on mineral uptake and growth of pepper and cucumber. Plant Soil Environ., 52 (3): 130-136. https://doi: 10.17221/3356-PSE

Haque, M.N., M.H. Ali and T.S. Roy, 2018. Specific gravity, dry matter and starch concentration of different potato cultivars as affected by arsenic contamination. Potato Research. https://doi.org/10.1007/s11540-017-9351-2.

Imas, P. and B. Sheva. 2000. Integrated nutrient management for sustaining crop yields in calcareous soils. Presented at GAU-PRII-IPI National Symposium on: Balanced nutrition of groundnut and other field crops grown on calcareous soils of India. September 19- 22, 2000. Junagadh, Gujarat, India.

Jackson, T.L., 1973. Soil Chemical Analysis. Prentice-Hall of India Private Limited. New Delhi.

James, L.G., 1988. Principles of farm irrigation systems design. Washington State University, 543.

Jatav, M.K., M. Kumar, S.P. Trehan, V.K. Dua and S. Kumar, 2013. Effect of Nitrogen and varieties of potato on yield and agronomic N use efficiency in North-Western plains of India". Potato Res., 40: 55-59.

Jehangir, I. A., M.A. Mir, M.A. Bhat and M.A. Ahangar, 2017. Biofertilizers an approach to sustainability in agriculture: a review. International Journal of Pure and Applied Bioscience, 5 (5): 327-334. http://dx.doi.org/10.18782/2320-7051.5011

Jen-Hshuan, C., 2006. The combined use of chemical and organic fertilizers and/or biofertilizer for crop growth and soil fertility. International workshop on Sustained Management of the SoilRhizosphere System for Efficient Crop Production and Fertilizer Use 16-20:1-10

Jochum, M.D., K.L. McWilliams, E.J. Borrego, M.V. Kolomiets, G. Niu, E.A. Pierson and Y.-K. Jo, 2019. Bioprospecting Plant Growth-Promoting Rhizobacteria That Mitigate Drought Stress in Grasses. Front. Microbiol. 10:2106. doi: 10.3389/fmicb.2019.02106.

Ju, I., B.Wj, S. Md, O. Ia and E. Oj, 2018. A review: biofertilizer; a key player in enhancing soil fertility and crop productivity. J. Microbiol. Biotechnol. Rep. 2(1): 22-28.

Kaur, M.N. and J.K. Chawla, 2005. Irrigation and potassium management in trickle fertigated potato (Solanum tuberosum L.). Indian Journal of Agricultural Science, 75 (5): 290-292.

Kawthar, A.E.R., S.M. Selim and S.A. Nasr, 2002. Nitrate and nitrite accumulation in potato tubers in relation to mineral nitrogen and biofertilization. Egypt Annals Agric. Sci., 47(1):107- 122.

Khan, A., R.U. Khan, Z. Khan, F.H.A. Khan and S.U. Shah, 2017. Effect of humic substances alone and in combination with micronutrients on potato yield and nutrients status, Int. J. Agron. Agri. 11(5): 24-29.

Khosro, M and S. Yousef, 2012. Bacterial bio-fertilizers for sustainable crop production: A review APRN Journal of Agricultural and Biological Science, 7(5):237-308.

Klute, A., 1986. Methods of Soil Analysis. Part 1. Physical and Mineralogical Methods", 2nd ed., American Society of Agronomy-Soil Science Society of America, Madison, Wisconsin, USA.

Leszczyński, W., 2004. Resistant starch - classification, structure, production. Pol. J. Food Nutr. Sci., 13(54), SI, 1: 37-50.

Lisińska, G., 2000. Raw material and technological factors shaping the quality of potato products. Scientific Conference Materials. Potato intended for consumption and industrial use and its processing (in Polish). Polanica Zdrój, November 8th, 51-59. 
Mahendran, P.P., N. Kumar and S. Saraswathy, 1996. Studies on the effect of biofertilizers on potato (Solanum tuberosum). South Indian Hort., 44 (3-4): 79-82.

Mahmoud. A.R. and M.M. Hafez, 2010. Increasing productivity of potato plants (Solanum tuberosum, L) by using potassium fertilizer and humic acid application. International Journal of Academic Research, 2 (2):83-88.

Malboobi, M.A. and P. Owlia et al., 2009. Solubilization of organic and inorganic phosphates by three highly efficient soil bacterial isolates. World J. Microbiol Biotechnol., 25(8):1471-1477.

Mamnabi, S., S. Nasrollahzadeh, K. Ghassemi-Golezani and Y. Raei, 2020. Improving yield-related physiological characteristics of spring rapeseed by integrated fertilizer management under water deficit conditions. Saudi Journal of Biological Sciences, 27: 797-804.

DOI: https://doi.org/10.1016/j.sjbs.2020.01.008.

Marschner, H., 1995. Mineral Nutrition of Higher Plants. $2^{\text {nd }}$ Edition. Academic Press, London.

Mfilinge, A., K. Mtei and N. Dakidemi, 2014. Effect of Rhizobium inoculation and supplementation with phosphorus and potassium on growth leaf chlorophyll content and nitrogen fixation of bush bean varieties. American Journal of Research Communication, 2(10):49-87.

Mohammadi, G.R., A. Rostami Ajirloo, M.E. Ghobadi and A. Najaphy, 2013. Effects of non-chemical and chemical fertilizers on potato (Solanum tuberosum L.) yield and quality. Journal of Medicinal Plants Research, 7(1): 36-42. https://doi: 10.5897/JMPR11.1638.

Mohammadi, K. and Y. Sohrabi, 2012. Bacterial biofertilizers for sustainable crop production: a review. ARPN Journal of Agricultural and Biological Science. 7(5): 307-316.

Molden, D., 1997. Accounting for water use and productivity. SWIM Paper 1. International irrigation Management Institute, Colombo, Sri Lanka.

Mosa A.A., 2012. Effect of the Application of Humic Substances on Yield, Quality and Nutrient Content of Potato Tubers in Egypt. In: He Z., Larkin R., Honeycutt W. (eds) Sustainable Potato Production: Global Case Studies. Springer, Dordrecht. http://doi-org-443.webvpn.fjmu.edu.cn/10.1007/978-94-007-4104-1_27

Nadeem, S.M., M. Ahmad, Z. Zahir, A. Javaid and M. Ashraf, 2014. The role of mycorrhizae and plant growth promoting rhizobacteria (PGPR) in improving crop productivity under stressful environments. Biotechnology Advances, 32(2): 429-448. https://doi.org/10.1016/j.biotechadv.2013.12.005.

Norman, Q.A, A.E. Clive, B. Peter, D.M. James, L. Stephen and W. Christie, 2003. Effects of vermicomposts on growth and marketable fruits of field-grown tomatoes, peppers and strawberries. Pedobiologia, 47:731-735.

Obidiegwu, J.E., G.J. Bryan, H.G. Jones and A. Prashar, 2015. Coping with drought: stress and adaptive responses in potato and perspectives for improvement. Front. Plant Sci. 6:542. doi: $10.3389 /$ fpls.2015.00542.

Onder, S. et al., 2005. Different irrigation methods and water stress effects on potato yield and yield components. Agricultural Water Management, 73 (1): 73-86.

Opena G.B. and G.A. Porter, 1999. Soil management and supplemental irrigation effects on potato: II. Root growth. Agron. J., 91:426-431

Page, A.L., R.H. Miller and D.R. Keeney, 1982. Methods of Soil Analysis Part 2: Chemical and Microbiological Properties", 2nd ed., Amer. Soc. of Agron., Madison, Wisconsin, USA.

Pagter, M., C. Bragato and H. Brix 2005.Tolerance and physiological responses of Phragmites australis to water deficit. Aquatic Botany, 81: 285-299

Parkinson, J.A. and S.E. Allen, 1975. A wet oxidation procedure suitable for the determination of nitrogen and mineral nutrients in biological materials. Commun. Soil Sci. and Plant Analysis, 6: $1-11$.

Pino, M.T., J.S. Skinner, E.J. Park, Z. Jeknic, P.M. Hayes, M.F. Thomashow and T.H.H. Chen, 2007. Use of a stress inducible promoter to drive ectopic AtCBF expression improves potato freezing tolerance while minimizing negative effects on tuber yield. Plant Bio-technology, 5: 591-604.

Ranganna, S., 1977. Manual of analysis of fruit and vegetable products. Central Food Technological Research Institute Mysore.

Rokhzadi, A., A. Asgharzadeh, F. Darvish, G. Nourmohammadi and E. Majidi, 2008. Influence of plant growth-promoting rhizobacteria on dry matter accumulation and yield of chickpea (Cicer arietinum L.) under field condition. Am-Euras. J. Agric. Environ. Sci. 3(2): 253-257. 
Rubiez, I.G., J.L. Stroehlein and N.F. Oebeker, 1991. Effect of irrigation method on urea phosphate reactions in calcareous soils. Commun. Soil Sci. Plant Anal., 22:431-435.

Saneoka, H., R.E.A. Moghaieb, G.S. Premachandra, K. Fujita, 2004. Nitrogen nutrition and water stress effects on cell membrane stability and leaf water relations in AgrostispalustrisHuds. Environ. Exp. Bot., 52: 131-138.

Scalenghe, R., A.C. Edwards and E. Barberis, 2012. Agricultural soil under a continental temperature climate susceptible to episodic reducing conditions and increased leaching of phosphorus. Journal of Environmental Management. (97):141-7.

Silva, E.C.D., R. J. M. C. Nogueira, M.A.D. Silva and M.B.D. Albuquerque, 2011. Drought Stress and Plant Nutrition. Plant Stress 5 (Special Issue 1), 32-41 (C2011 Global Science Books

Singh, V., K. Priyanka and D. Singh, 2011. Use of plant growth promoting rhizobacteria in crop production.Indian Farming, 61 (2): 12-16.

Šircelj, H., M. Tausz, D. Grill and F. Batić, 2005. Biochemical responses in leaves of two apple tree cultivars subjected to progressing drought. Journal of Plant Physiology, 162: 1308-1318

Smith, N., 1991. CROPWAT model for ETo calculation using penman monteith method. FAO.

Soil Survey Staff, 2010. Keys to soil taxonomy, $11^{\text {th }}$ edn. USDA-Natural Resources Conservation Services, Washington, DC.

Stancheva, I., I. Dimitrov, N. Kaloyanova, N. Dinev and N. Poushkarov, 1995. Improvement of the nitrogen uptake and nitrogen content in maize (Zea mays) by inoculation with Azospirillum brasilense. Agrochimica, 39: 299-306.

Stark J.C., D.T. Westermann and B.G. Hopkins, 2004. Nutrient management guidelines for Russet Burbank potatoes. Bulletin 840. Moscow, ID: CALS University of Idaho.

Steel, R.G.D. and J.H. Torrie, 1982. Principals and Procedures of Statistics. A Biometrical Approach. Mc Graw Hill Book Company, New York. USA.

Suhag, M., 2016. Potential of biofertilizers to replace chemical fertilizers. Int. Adv. Res. J. Sci. Eng. Technol., 3: 163-167.

Taalab, A.S., G.W. Ageeb, H.S. Siam and S.A. Mahmoud, 2019. Some characteristics of calcareous soils. A review. Middle East Journal of Agriculture Research. 8 (1):96-105.

Van-Schaik, A.H., P.C. Struik and T.G. Damian, 1997. Effects of irrigation and N on the vegetative growth of Aloe barbadensis Mill. in Aruba. Trop. Agric., 74: 104-109.

Vinković, T., N. Parađiković, M. Tkalec, T. Teklić and Z. Lončarić, 2012. Effect of biostimulants on nutrient content in some organs and tomato plant. In M. Pospišil (Ed.) Book of abstract of 47th Croatian and 7th International Symposium on Agriculture, 2012 February 13-17, Faculty of agronomy, University of Zagreb, pp. 114-115. http://sa.agr.hr/pdf/2012/sa2012_a0417.pdf.

Wahba, M.M, F. Labib and A. Zaghloul, 2019. Management of calcareous soils in arid region (Review Paper). Int. J. of Environmental Pollution \& Environmental Modelling, 2(5): 248-258.

Yang, J., J.W. Kloepper and C.M. Ryu, 2009. Rhizosphere bacteria help plants tolerate abiotic stress. Trends Plant Sci. 14: 1-4.

Yaseen, R., J. Shafi, W. Ahmad, M.S. Rana, M. Salim and S.A. Qaisrani, 2014. Effect of deficit irrigation and mulch on soil physical properties, growth and yield of maize. Environ. Ecol. Res., 2: $122-137$.

Yuan, B.-Z., S. Nishiyama and Y. Kang, 2003. Effects of different irrigation regimes on growth and yield of drip-irrigated potato. Agricultural Water Management, 63 (3): 153-167.

Yuncong, Li., 2001. Calcareous soils in Miami-Dade county. Fact. Sheet SL 183, a publication of the Soil and Water Science Department, Florida Cooperative Extension Service, Institute of Food and Agricultural Sciences, University of Florida. (C.F. EDIS website at: http:// edis.ifas.ufl.edu).

Zaghloul, R.A., 2002. Biofertilization and organic manuring efficiency on growth and yield of potato plants (Solanum tuberosum L.). In: Recent Technologies in Agriculture. Proceedings of the $2^{\text {nd }}$ Congress. Fac. Agric., Cairo Univ., 28-30 October, 79-95.

Zahir, A., M. Arshad and W. Frankenberger, 2004. Plant growth promoting rhizobacteria: applications and perspectives in agriculture. Advances in Agronomy, 81: 97-168.

Zgórska, K. and A. Frydecka-Mazurczyk, 2002. Standards and quality requirements of edible potato and potato intended for food processing. In: Production and market of edible potato (in Polish) (Ed. J. Chotkowski). Wieś Jutra, 183-192. 